\title{
Energy-Spectrum Efficient Content Distribution in Fog-RAN Using Rate-Splitting, Common Message Decoding, and 3D- Resource Matching
}

This paper was downloaded from TechRxiv (https://www.techrxiv.org).

\section{LICENSE}

CC BY 4.0

SUBMISSION DATE / POSTED DATE

$09-09-2020 / 12-01-2021$

\section{CITATION}

HASSAN, MD ZOHEB (2020): Energy-Spectrum Efficient Content Distribution in Fog-RAN Using RateSplitting, Common Message Decoding, and 3D-Resource Matching. TechRxiv. Preprint. https://doi.org/10.36227/techrxiv.12933761.v2

$\mathrm{DOI}$ 


\title{
Energy-Spectrum Efficient Content Distribution in Fog-RAN Using Rate-Splitting, Common Message Decoding, and 3D-Resource Matching
}

\author{
Md. Zoheb Hassan, Md. Jahangir Hossain, Senior Member, IEEE, \\ Julian Cheng, Senior Member, IEEE, and Victor C. M. Leung, Life Fellow, IEEE
}

\begin{abstract}
Multi-objective resource allocation is studied for edge-caching enabled fog-radio access network. Notably, joint maximization of the energy-efficiency (EE) and spectrum-efficiency (SE) and interference management are investigated for distributing contents from the cache-enabled fog access points (F-APs) and cloud base station (CBS) to the user devices (UDs). In our envisioned system, the UDs are grouped into multiple non-overlapping device-clusters based on their locations. A rate-splitting with common message decoding based transmission strategy is applied to enable UDs of each device-cluster to receive data from a suitably selected F-AP and CBS over the same radio resource blocks. To maximize system EE and SE jointly, a multi-objective optimization problem (MOOP) is formulated and it is solved in three stages. At first, by employing the $\epsilon$-constraint method, the MOOP is converted to an EE-SE trade-off optimization problem. Then, by leveraging iterative function evaluation based power control and generalized 3D-resource matching, the EE-SE trade-off optimization problem is solved and a novel resource allocation algorithm is proposed to obtain near-optimal Pareto-front for the proposed MOOP. To reduce the complexity of obtaining near-optimal Pareto-front, a sub-optimal resource allocation algorithm is proposed as well. Finally, a low-complexity algorithm is devised to select a suitable operating EE-SE pair from the obtained Pareto-front. The conducted simulations demonstrate that the proposed resource allocation schemes achieve substantial improvement of system EE and SE over the benchmark schemes.
\end{abstract}

\section{INTRODUCTION}

Leveraging centralized cloud processing based resource allocation, distributed signal processing, and popular content caching at the network edge, fog-radio access network (F-RAN) presents a revolutionary paradigm to satisfy the beyond $5 \mathrm{G}(\mathrm{B} 5 \mathrm{G})$ performance requirements [1]. In edgecaching (EC) enabled F-RAN, the content distribution phase is significantly important to ensure throughput and diverse latency requirements of the user devices (UDs). Particularly, EC enabled F-RAN jointly exploits both the cache-enabled fog access points (F-APs) and centralized cloud

Md. Zoheb Hassan, Md. Jahangir Hossain and Julian Cheng are with School of Engineering, The University of British Columbia, Kelowna, BC, Canada (e-mail: zohassan@mail.ubc.ca, \{jahangir.hossain, julian.cheng\}@ubc.ca). Victor C. M. Leung is with the College of Computer Science, Shenzhen University, Shenzhen 518060, China, and also with the Department of Electrical and Computer Engineering, The University of British Columbia, Vancouver, Canada (e-mail: vleung@ieee.org). 
base station (CBS) to deliver contents to the UDs [2]. Hence, well-designed resource allocation plays a vital role for improving the content distribution performance of EC enabled F-RAN.

Because of the proliferation of mobile devices and various applications in the B5G network, there is a dire need of solution to efficiently deliver high-volume and latency-sensitive enhanced mobile broadband (eMBB) data, generated from 3D video streaming and extended reality, to the UDs. Using the joint edge-cloud processing, EC enabled F-RAN presents a promising architecture to deliver eMBB data to the UDs with reduced latency [3]. However, the advantage of F-RAN enabled eMBB data delivery is confronted by the increased interference in the dense network and scarcity of the spectrum resources. In particular, interference severely reduces the spectrum-efficiency (SE) which is detrimental to the eMBB data. The conventional approach of interference management by scheduling the active links over orthogonal spectrum resources strictly limits number of the supported UDs in EC enabled F-RAN, and thus, it is incapable to satisfy the data demand of the overly congested B5G network. A promising way to improve the spectrum resource utilization is to group the UDs into multiple device-clusters where the members of each cluster are scheduled to receive data over the same spectrum resources. However, clustering inevitably increases the interference in the network and requires a complex interference management scheme. In addition, the number of F-APs in F-RAN needs to be increased to satisfy enhanced data-rate requirements of a large number of UDs. Hence, from a network operation point of view, maximization of the system energy-efficiency (EE) is also imperative to ensure sustainable operation of F-RAN. Indeed, the B5G network is expected to have 10 times enhancement of EE than 5G network [4]. Accordingly, F-RAN enabled eMBB data delivery calls for a novel solution that maximizes system EE and SE jointly, and provides efficient interference management with improved spectrum resource utilization. However, developing such a solution is non-trivial. This is because on one hand, joint maximization of EE and SE requires to maximize two conflicting objectives, and on the other hand, interference management in a network with multiple device-clusters is a well-known NP-hard problem. To address these challenges, we focus on designing novel resource allocation scheme while aiming at joint maximization of system EE and SE and interference management for the content distribution phase of EC enabled F-RAN.

\section{A. Related Works}

EC significantly reduces the backhaul energy consumption and leads to improved EE [5]. In EC enabled system, the enhancement of $\mathrm{EE}$ is attributed by optimizing both the content placement and content distribution phases [6]. A large number of recent works have investigated on-device caching and improvement of the system EE by capitalizing the proximity gain of an 
optimized device-to-device (D2D) assisted content distribution phase. Using game theory, the authors in [8] designed optimized caching strategy and incentive mechanism to encourage the devices to participate in the EC system. The authors in [9] proposed optimized proactive caching strategy and transmission power control to offload the contents from the centralized content server via D2D links while reducing energy consumption of the offloading process. The authors in [10] proposed a novel D2D-clustering based content distribution framework for improving EE of the network. In [11], novel unicast and multicast based D2D-assisted content distribution schemes were proposed for improving EE of network. Using the tools from stochastic geometry, the authors obtained optimal caching strategy for D2D-clustering enabled network [12]. Besides device-caching and D2D assisted content distribution, multi-level EC was also utilized to improve EE of the system. In [13], [14], [39], the authors studied multi-level EC enabled wireless network where mode selection, transmission power, and file combination were optimized to reduce energy consumption of the content distribution phase. Although the aforementioned works depicted the advantages of EC for improving system EE, these works did not explore interference management for delivering the cached contents. In the most of these works, interference was treated as a noise and consequently, the resultant EE is sub-optimal. Hence, it is imperative to investigate the benefit of interference management for improving EE of EC enabled system.

Resource allocation is envisaged to play the pivotal role for interference management in EC enabled F-RAN. In the contemporary literature, non-orthogonal multiple access (NOMA) has received strong reputation to multiplex multiple UDs over the same spectrum resource(s), and consequently, NOMA has also been applied to manage interference in F-RAN. By optimizing power control and spectrum resource-UD matching, the authors in [16]-[18] developed several novel resource allocation schemes to manage interference and maximize SE of NOMA-based FRAN. The existing literature also focused on improving EE of F-RAN through resource allocation and interference management. The authors in [19] developed cross-tier interference aware pricing mechanism for two-tier F-RAN, and developed a game theoretic resource allocation scheme. Considering the cost of content caching at the F-APs, the authors in [20] developed optimized beamforming vectors and UD/F-AP associations to maximize a cost-aware EE of F-RAN. The optimized beamforming vectors and UD/F-AP associations were also developed to maximize EE of F-RAN while considering outdated channel information at the cloud [21]. Note that the aforementioned works solely concentrated on optimizing either SE or EE of F-RAN in an individual manner. However, optimization of only SE is insufficient to characterize energy saving in the network, and optimization of only EE does not necessarily lead to an optimized SE. Hence, 
to ensure both greenness for network operation and improved SE, as required by B5G network, optimization of the trade-off between EE and SE of F-RAN is inherently important.

Rate-splitting with common message decoding (RS-CMD) has recently been emerged as a robust and resource efficient transmission strategy for mitigating interference [22]. RS-CMD enables decoding/canceling partial interference and treating partial interference as a noise, and thus, it appears as a more generalized multi-user transmission strategy than NOMA [23]. The problem of SE optimization for RS-CMD system was extensively studied for various wireless networking scenarios, such as, multi-user broadcast system [24], cloud-radio access network [25], cooperative network with user relaying [26], and aerial network [27]. Moreover, both EE maximization and EE-SE trade-off optimization for RS-CMD systems were studied in [28]-[30]. Although the aforementioned works applied RS-CMD in multi-antenna setting, RS-CMD is also advantageous for interference management in single-antenna network [31]. Motivated by the success of RS-CMD in interference management, in this work, RS-CMD is employed for the content distribution phase of EC enabled F-RAN. Note that, for an RS-CMD integrated system, the aforementioned works investigated a downlink scenario with single transmitter and multiple receivers and did not consider the problem of association between receivers and transmitters. However, the association between UDs and F-APs is crucial in EC enabled F-RAN as mentioned in [3], [17]. Consequently, in this work, we optimize the EE-SE trade-off of RS-CMD integrated F-RAN while taking the problem of association between UDs and F-APs into account.

\section{B. Contributions and Paper Organization}

In this work, a novel cluster-based resource allocation framework is developed for EC enabled F-RAN. In our envisioned system, the UDs are clustered based on their locations. We employ RS-CMD based transmission strategy to enable UDs of each device-cluster to receive data from a suitably selected cache-enabled F-AP and CBS over the same radio resource blocks (RRBs). Accordingly, our envisioned system enhances utilization of the limited RRBs in the content distribution phase of EC enabled F-RAN. However, the considered strategy leads to increased interference that affects both system EE and SE. To this end, our developed resource allocation framework aims to efficiently manage interference in the content distribution phase of EC enabled F-RAN while jointly maximizing system EE and SE. To the best of authors' knowledge, this is the first work that optimizes the trade-off between system EE and SE for the RS-CMD integrated and EC enabled F-RAN. The specific contributions of this work are summarized as follows.

- A multi-objective optimization problem (MOOP) is formulated to maximize the EE and SE of the system jointly. Notably, we optimize the power allocation of the F-APs and 
CBS, association between F-APs and device-clusters, and allocation of the RRBs among the device-clusters. To obtain the near-optimal Pareto-front of the proposed MOOP, it is converted to an EE-SE trade-off optimization problem by applying the $\epsilon$-constraint method. Since the EE-SE trade-off optimization problem is NP-hard, a three stage solution is devised.

- At the first stage, we determine the maximum achievable system EE and SE by decomposing the resource allocation into two sub-problems. The first sub-problem obtains near-optimal power allocations of the CBS and F-APs by applying an iterative function evaluation (IFE) approach. The second sub-problem obtains a Pareto-efficient matching of RRBs and F-APs with the device-clusters by solving a generalized 3D-resource matching problem. Based on a block-alternating accent method, two convergent algorithms of polynomial computational complexity are proposed to determine the maximum achievable EE and SE of the system.

- At the second stage, we propose a novel iterative rate-splitting and resource-matching (IRSRM) algorithm to solve the EE-SE trade-off optimization problem iteratively. I-RSRM obtains the near-optimal Pareto-front of the proposed MOOP by iteratively adjusting the trade-off between EE and SE. To reduce the complexity of achieving near-optimal Paretofront of the proposed MOOP, a sub-optimal resource allocation algorithm, referred as, decomposed rate-splitting and resource-matching (D-RSRM), is proposed as well.

- At the final stage, a low-complexity algorithm is developed to select an operating EE-SE pair from the solutions obtained by I-RSRM and D-RSRM algorithms. The developed algorithm provides a suitable trade-off between system EE and SE while adjusting a single parameter. Extensive simulations are conducted to verify the superiority of the proposed near-optimal and sub-optimal resource allocation algorithms over several benchmark schemes.

The rest of the manuscript has following organizations. Section II provides overall system model and problem formulation. Maximum achievable EE and SE of the system are obtained in Section III. EE-SE trade-off optimization and the proposed algorithms are discussed in Section IV. Simulation results and concluding remarks are presented in Sections V and VI, respectively.

\section{SySTEM MODEL}

\section{A. System Overview}

Consider a downlink F-RAN with one CBS, $L$ single antenna F-APs, $N$ orthogonal RRBs, and $M$ single antenna UDs. Fig. 1 illustrates an example of such a network. Here, F-APs are connected with the CBS via fronthaul links. As per the contemporary F-RAN literature [16], [17], CBS proactively places data of the UDs at the cache-memory of F-APs in offline (i.e., before the communication takes place). As such, in online the F-APs can directly transmit data to the UDs 


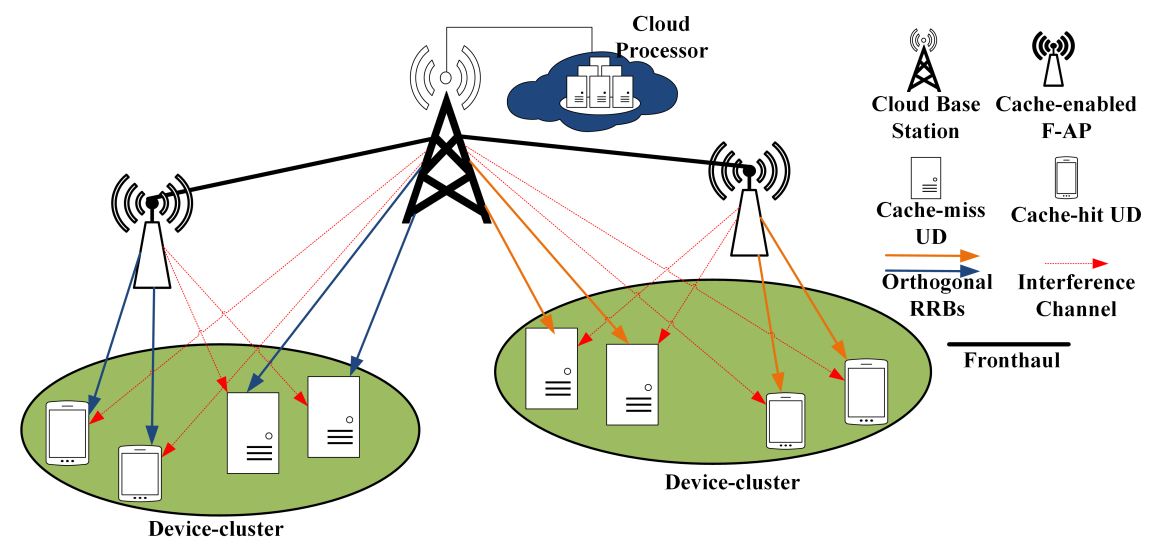

Fig. 1: Downlink transmission in F-RAN with 2 cache-enabled F-APs, 2 RRBs, and 8 UDs.

without requiring data to be delivered from CBS over fronthaul links. The considered system is a standard F-RAN architecture [2], and it is benefited from both the cloud processing empowered resource allocation and local signal processing/encoding operations of F-APs. For simplicity, we assume that each F-AP caches identical contents. Let $\mathcal{N}=\{1,2, \cdots, N\}, \mathcal{L}=\{1,2, \cdots, L\}$ and $\mathcal{M}=\{1,2, \cdots, M\}$ be the set of available orthogonal RRBs, F-APs, and UDs, respectively.

Based on the locations of UDs, multiple non-overlapping device-clusters are formed as such the mean-square distance between the clustered UDs and cluster center is minimized. Let, $M$ UDs be grouped into $K$ device-clusters, denoted by, $\mathcal{S}_{1}, \mathcal{S}_{2}, \cdots, \mathcal{S}_{K}$ where $\mathcal{S}_{k} \cap \mathcal{S}_{k^{\prime} \neq k}=$ $\varnothing, \forall k, k^{\prime} ; \cup_{k=1}^{K} \mathcal{S}_{k}=\mathcal{M}$. Since the F-APs are cost-efficient devices, they can cache only a subset of the UDs' data in their storage. To this end, each device-cluster is further decomposed into two non-overlapping sets of cache-hit and cache-miss UDs. The requested data of the cache-hit UDs is available at the F-APs, and hence, they obtain data by directly accessing the local cache. In contrast, the requested data of the cache-miss UDs are not available at the F-APs, and as a result, they can obtain the data only from CBS. Note that our proposed resource allocation framework is valid for any device-clustering algorithm. Hence, in the ensuing analysis, we consider that the device-clusters are given, and we focus on the resource allocations among the device-clusters.

In a given device-cluster, both the cache-hit and cache-miss UDs receive data from a certain F-AP and CBS, respectively, over the same RRBs. Hence, the system EE and SE depends on the mitigation of the following interference in the content distribution phase ${ }^{1}$, namely, (i) inter device-cluster interference, (ii) interference between F-AP and CBS in each device-cluster, (iii) intra device-cluster interference, such as, interference among the cache-hit UDs and interference among the cache-miss UDs in each device-cluster. To combat inter device-cluster interference,

\footnotetext{
${ }^{1}$ Interference from the malicious and illegal devices also affects the system reliability. However, mitigation of such interference is beyond the scope of this work, and it will be considered in the future extension of this work.
} 
each device-cluster is allocated with certain orthogonal RRBs. To combat interference between F-AP and CBS in a given device-cluster, joint F-AP and CBS power allocation over the shared RRBs is leveraged. Finally, to combat intra device-cluster interference, 1-layer RS-CMD is exploited for both the cache-hit and cache-miss UDs in each device-cluster. Along the lines of [3], [17], each device-cluster is allowed to be associated with maximum one F-AP. Meanwhile, an F-AP can serve multiple device-clusters over the orthogonal RRBs.

For analytical tractability, the following assumptions are made. A1: The described system runs on a slotted-time basis where the overall time duration is divided into equal and non-overlapping time-slots (TSs). We assume block fading channel where the channels remain constant in a given TS, and can independently vary in different TSs. Data transmission from all the F-APs at each TS is synchronized by the CBS. A2: UDs can accurately perform successive interference cancellation (SIC), and the locations of UDs remain fixed for a number of TSs. A3: F-AP and CBS has accurate knowledge of the local and global channel state information, respectively, of both cache-hit and cache-miss UDs in the system. A4: Using the cloud processing, all the resource allocation tasks are executed at the CBS in a centralized manner. Note that the aforementioned assumptions are common in the contemporary literature of F-RAN [2], [16], [17]. Because of these assumptions, the proposed work provides the maximum theoretical performance gain for EC enabled F-RAN by jointly optimizing power allocation of the F-APs and CBS, association between F-APs and device-clusters, and allocation of the RRBs among the device-clusters.

\section{B. RS-CMD Based Transmission Strategy}

We describe RS-CMD based transmission strategy for the $j$-th device-cluster, $\mathcal{S}_{j}$. The cachehit and cache-miss UDs of the $j$-th device-cluster are denoted by $\mathcal{S}_{j}^{(L)}$ and $\mathcal{S}_{j}^{(H)}$, respectively, where $\mathcal{S}_{j}^{(L)} \cap \mathcal{S}_{j}^{(H)}=\varnothing$ and $\mathcal{S}_{j}^{(L)} \cup \mathcal{S}_{j}^{(H)}=\mathcal{S}_{j}$. Here, $\mathcal{S}_{j}^{(L)}=\left\{1,2, \cdots,\left|\mathcal{S}_{j}^{(L)}\right|\right\}$ and $\mathcal{S}_{j}^{(H)}=$ $\left\{1,2, \cdots,\left|\mathcal{S}_{j}^{(H)}\right|\right\}$ where $|\cdot|$ refers to the cardinality of a set. We consider that the $l$-th F-AP and the $n$-th RRB are assigned to the $j$-th device-cluster. Hence, the UDs in the $\mathcal{S}_{j}^{(L)}$ and $\mathcal{S}_{j}^{(H)}$ sets receive data from the $l$-th F-AP and CBS, respectively, over the $n$-th RRB. The $l$-th FAP first splits the data of the $e$-th UD into two parts, denoted by, $\left\{\mathcal{W}_{e^{(c)}}, \mathcal{W}_{e^{(p)}}\right\}, \forall e \in \mathcal{S}_{j}^{(L)}$. Subsequently, the $l$-th F-AP combines and encodes $\mathcal{W}_{e(c)}, \forall e \in \mathcal{S}_{j}^{(L)}$ into a common message denoted by $s_{c}$. Simultaneously, the $l$-th F-AP encodes $\mathcal{W}_{e}(p), \forall e \in \mathcal{S}_{j}^{(L)}$, into $\left|\mathcal{S}_{j}^{(L)}\right|$ private messages denoted by $\left\{s_{e}^{(p)}\right\}$. The signal transmitted by the $l$-F-AP over the $n$-th RRB is expressed as $X_{l, n}=\sqrt{P_{l, n}^{C, j}} s_{c}+\sum_{e=1}^{\left|\mathcal{S}_{j}^{(L)}\right|} \sqrt{P_{l, e, n}^{p, j}} s_{e}^{(p)}$ where $P_{l, n}^{C, j}$ and $\left\{P_{l, e, n}^{p, j}\right\}$ denote the transmission powers assigned for the common and private messages for the cache-hit UDs in the $j$-th device-cluster, respectively. Similarly, CBS encodes the data of the cache-miss UDs into a common message, 


$$
\begin{aligned}
& \gamma_{l, n}^{C, j}=\frac{P_{l, n}^{C, j}\left|h_{k_{j}, n}^{(l)}\right|^{2}}{\left(\sum_{e=1}^{\left|\mathcal{S}_{j}^{(L)}\right|} P_{l, e, n}^{p, j}\right)\left|h_{k_{j}, n}^{(l)}\right|^{2}+\left(Q_{n}^{C, j}+\sum_{t=1}^{\left|\mathcal{S}_{j}^{(H)}\right|} Q_{t, n}^{p, j}\right)\left|h_{k_{j}, n}^{(0)}\right|^{2}+\sigma^{2}}, \\
& \widetilde{\gamma}_{n}^{C, j}=\frac{Q_{n}^{C, j}\left|h_{\tilde{k}_{j}, n}^{(0)}\right|^{2}}{\left(\sum_{t=1}^{\left|\mathcal{S}_{j}^{(H)}\right|} Q_{t, n}^{p, j}\right)\left|h_{\tilde{k}_{j}, n}^{(0)}\right|^{2}+\left(P_{l, n}^{C, j}+\sum_{e=1}^{\left|\mathcal{S}_{j}^{(L)}\right|} P_{l, e, n}^{p, j}\right)\left|h_{\tilde{k}_{j}, n}^{(l)}\right|^{2}+\sigma^{2}} .
\end{aligned}
$$

denoted by, $\tilde{s}_{c}$, and $\left|\mathcal{S}_{j}^{(H)}\right|$ private messages, denoted by, $\left\{\tilde{s}_{t}^{(p)}\right\}$. The signal transmitted by the CBS over the $n$-th RRB is expressed as $\tilde{X}_{n}=\sqrt{Q_{n}^{C, j}} \tilde{s}_{c}+\sum_{t=1}^{\left|\mathcal{S}_{j}^{(H)}\right|} \sqrt{Q_{t, n}^{p, j}} \tilde{s}_{t}^{(p)}$ where $Q_{n}^{C, j}$ and $\left\{Q_{t, n}^{p, j}\right\}$ denote the transmission powers assigned for the common and private messages for the cache-miss UDs in the $j$-th device-cluster, respectively. The received signals at the UDs are expressed as

$$
\begin{aligned}
& Y_{e}^{(n)}=h_{e, n}^{(l)} X_{l, n}+h_{e, n}^{(0)} \tilde{X}_{n}+n, \forall e \in \mathcal{S}_{j}^{(L)} \\
& \tilde{Y}_{t}^{(n)}=h_{t, n}^{(0)} \tilde{X}_{n}+h_{t, n}^{(l)} X_{l, n}+n, \forall t \in \mathcal{S}_{j}^{(H)}
\end{aligned}
$$

where $h_{e, n}^{(l)}\left(h_{t, n}^{(l)}\right)$ is the channel gain between the $l$-th F-AP and the $e$-th UD (the $t$-th UD) over the $n$-th RRB; $h_{e, n}^{(0)}\left(h_{t, n}^{(0)}\right)$ is the channel gain between CBS and the $e$-th UD (the $t$-th UD) over the $n$-th RRB; and $n$ is the additive white Gaussian noise with variance $\sigma^{2}$.

The UDs in the $\mathcal{S}_{j}^{(L)}$ and $\mathcal{S}_{j}^{(H)}$ sets first decode the common messages transmitted from the $l$-th F-AP and CBS, respectively. All the UDs of the $\mathcal{S}_{j}^{(L)}$ and $\mathcal{S}_{j}^{(H)}$ sets should be able to decode their corresponding common messages. We denote $k_{j}=\arg \min _{e \in \mathcal{S}_{j}^{(L)}} \frac{\left|h_{e, n}^{(l)}\right|^{2}}{\left(Q_{n}^{C, j}+\sum_{t=1}^{\left|\mathcal{S}_{j}^{(H)}\right|} Q_{t, n}^{p, j}\right)\left|h_{e, n}^{(0)}\right|^{2}+\sigma^{2}}$ and $\tilde{k}_{j}=\arg \min _{t \in \mathcal{S}_{j}^{(H)}} \frac{\left|h_{t, n}^{(0)}\right|^{2}}{\left(P_{l, n}^{C, j}+\sum_{e=1}^{\left|\mathcal{S}_{j}^{(L)}\right|} P_{l, e, n}^{p, j}\right)\left|h_{t, n}^{(l)}\right|^{2}+\sigma^{2}}$. Consequently, for the common messages over the $n$-th RRB, the $l$-th F-AP and CBS target the achievable data rate of the UDs indexed by $k_{j}$ and $\tilde{k}_{j}$, respectively. The common message data rates for the cache-hit and cache-miss UDs are expressed as $R_{l, n}^{C, j}=\log _{2}\left(1+\gamma_{l, n}^{C, j}\right)$ and $\widetilde{R}_{n}^{C, j}=\log _{2}\left(1+\widetilde{\gamma}_{n}^{C, j}\right)$, respectively. Here, $\gamma_{l, n}^{C, j}$ and $\gamma_{n}^{C, j}$ are defined as (2) and (3), respectively, at the top of the current page.

After decoding the common messages, both the cache-hit and cache-miss UDs decode their own private messages while canceling the interference from the decoded common messages by applying SIC, and treating the private messages of other UDs in the system as noise. The cachehit (cache-miss) UDs also treat the interference from the common message of the cache-miss (cache-hit) UDs as noise. Thus, the scheduled data rates for the private messages of the $e$-th cache-hit and $t$-th cache-miss UDs, $\forall e \in \mathcal{S}_{j}^{(L)}$ and $\forall t \in \mathcal{S}_{j}^{(H)}$, are obtained as, respectively, 


$$
\begin{aligned}
R_{l, e, n}^{\boldsymbol{p}, j}=\log _{2}\left(1+\gamma_{l, e, n}^{\boldsymbol{p}, j}\right) \text { and } \widetilde{R}_{t, n}^{p, j}=\log _{2}\left(1+\widetilde{\gamma}_{t, n}^{\boldsymbol{p}, j}\right) \text {. Here, } \\
\gamma_{l, e, n}^{\boldsymbol{p}, j}=\frac{P_{l, e, n}^{p, j}\left|h_{e, n}^{(l)}\right|^{2}}{\left(\sum_{\substack{\tilde{\tilde{e}}=1 \\
\tilde{e} \neq e}}^{\left|\mathcal{S}_{j}^{(L)}\right|} P_{l, \tilde{e}, n}^{p, j}\right)\left|h_{e, n}^{(l)}\right|^{2}+\left(Q_{n}^{C, j}+\sum_{t=1}^{\left|\mathcal{S}_{j}^{(H)}\right|} Q_{t, n}^{p, j}\right)\left|h_{e, n}^{(0)}\right|^{2}+\sigma^{2}}, \\
\widetilde{\gamma}_{t, n}^{\boldsymbol{p}, j}=\frac{Q_{t, n}^{p, j}\left|h_{t, n}^{(0)}\right|^{2}}{\left(\sum_{\substack{\tilde{t}=1 \\
\tilde{t} \neq t}}^{\left|\mathcal{S}_{j}^{(H)}\right|} Q_{\tilde{t}, n}^{p, j}\right)\left|h_{t, n}^{(0)}\right|^{2}+\left(P_{l, n}^{C, j}+\sum_{e=1}^{\left|\mathcal{S}_{j}^{(L)}\right|} P_{l, e, n}^{p, j}\right)\left|h_{t, n}^{(l)}\right|^{2}+\sigma^{2}} .
\end{aligned}
$$

Using (2)-(5), we obtain the total scheduled data rates for the cache-hit and cache-miss UDs in the $j$-th device-cluster, respectively, as $\mathrm{R}_{l, n, j}^{(L)}=R_{l, n}^{C, j}+\sum_{e \in \mathcal{S}_{j}^{(L)}} R_{l, e, n}^{p, j}$ and $\mathrm{R}_{n, j}^{(G)}=\widetilde{R}_{n}^{C, j}+$ $\sum_{t \in \mathcal{S}_{j}^{(H)}} \widetilde{R}_{t, n}^{p, j}$. Therefore, the overall throughput of the $j$-th device-cluster, given that the $l$-th FUE and the $n$-th RRB are assigned to the $j$-th device-cluster, is obtained as, $\mathrm{T}_{j}^{(l, n)}=\mathrm{R}_{l, n, j}^{(L)}+\mathrm{R}_{n, j}^{(G)}$.

\section{Problem Formulation}

We introduce two binary variables, $a_{l, j} \in\{0,1\}$ and $b_{n, l, j} \in\{0,1\}$ such that $a_{l, j}=1$ if the $l$-th F-AP is associated with the $j$-th device-cluster and $a_{l, j}=0$ otherwise; and $b_{n, l, j}=1$ if the $n$-th RRB is assigned to both the $l$-th F-AP and the $j$-th device-cluster and $b_{n, l, j}=0$ otherwise. Hence, the total power consumption of the $l$-th F-AP is obtained as $\mathrm{P}_{l}=P_{c}+$ $\frac{1}{\varepsilon} \sum_{j=1}^{K} \sum_{n=1}^{N} a_{l, j} b_{n, l, j}\left(P_{l, n}^{C, j}+\sum_{e \in \mathcal{S}_{j}^{(L)}} P_{l, e, n}^{p, j}\right)$ where $P_{c}$ is the constant circuitry power consumption and $\varepsilon$ is the power amplifier efficiency. Meanwhile, the total power consumption of the CBS is obtained as $\mathrm{P}_{C B S}=P_{c}+\frac{1}{\varepsilon} \sum_{j=1}^{K} \sum_{l=1}^{L} \sum_{n=1}^{N} a_{l, j} b_{n, l, j}\left(Q_{n}^{C, j}+\sum_{t \in \mathcal{S}_{j}^{(H)}} Q_{t, n}^{p, j}\right)$. Thereby, the system EE and SE are obtained as, respectively, $\operatorname{EE}(\boldsymbol{P}, \boldsymbol{Q}, \boldsymbol{a}, \boldsymbol{b})=\frac{\sum_{j=1}^{K} \sum_{l=1}^{L} \sum_{n=1}^{N} a_{l, j} b_{n, l, j} \mathrm{~T}_{j}^{(l, n)}}{\sum_{l \in \mathcal{L}} \mathrm{P}_{l}+\mathrm{P}_{C B S}}$, and $\operatorname{SE}(\boldsymbol{P}, \boldsymbol{Q}, \boldsymbol{a}, \boldsymbol{b})=\sum_{j=1}^{K} \sum_{l=1}^{L} \sum_{n=1}^{N} a_{l, j} b_{n, l, j} \mathrm{~T}_{j}^{(l, n)}$. We formulate an optimization problem, given by $\mathrm{P} 0$ at the top of next page, to maximize both the system $\mathrm{EE}$ and $\mathrm{SE}$ by jointly performing transmission power allocation of the F-APs and CBS, association between the F-APs and deviceclusters, and allocation of RRBs among the device-clusters. In P0, C1 implies that each devicecluster will be associated with only one F-AP whereas a given F-AP can be associated with maximum $N_{R}$ device-clusters; C2 implies the orthogonal allocation of the RRBs among the device-clusters; $\mathrm{C} 3$ implies that the $n$-th RRB can be assigned to both the $l$-th F-AP and the $j$-th device-cluster if and only if the $l$-th F-AP is associated with the $j$-th device-cluster; and C4 provides the transmission power allocation constraints for F-AP and CBS with $P$ max and $P$ max ${ }_{\text {ax }}^{\mathrm{CBS}}$ as the maximum transmission power of the F-AP and CBS, respectively.

$\mathrm{P0}$ is a non-convex MOOP. The $\epsilon$-constraint method can obtain Pareto-optimal solution for a non-convex MOOP [32]. Consequently, we employ the $\epsilon$-constraint method to obtain a Pareto- 


$$
\begin{array}{ll}
\text { P0: } & \max _{\boldsymbol{P} \geq \mathbf{0}, \boldsymbol{Q} \geqq \mathbf{0},\{\mathbf{a}, \mathbf{b}\} \in\{0,1\}}\{\operatorname{EE}(\boldsymbol{P}, \boldsymbol{Q}, \boldsymbol{a}, \boldsymbol{b}), \operatorname{SE}(\boldsymbol{P}, \boldsymbol{Q}, \boldsymbol{a}, \boldsymbol{b})\} \\
\text { s.t. }\left\{\begin{array}{l}
\mathrm{C} 1: \quad \sum_{l=1}^{L} a_{l, j}=1 ; \sum_{j=1}^{K} a_{l, j} \leq N_{R}, \forall l \in \mathcal{L}, j \in\{1,2, \cdots, K\} \\
\mathrm{C} 2: \sum_{j=1}^{K} \sum_{l=1}^{L} b_{n, l, j}=1 ; \sum_{n=1}^{N} \sum_{l=1}^{L} b_{n, l, j} \geq 1, \forall n \in \mathcal{N}, l \in \mathcal{L}, j \in\{1,2, \cdots, K\} \\
\mathrm{C} 3: b_{n, l, j} \leq a_{l, j}, \forall n \in \mathcal{N}, l \in \mathcal{L}, j \in\{1,2, \cdots, K\} \\
\mathrm{C} 4: \mathrm{P}_{l} \leq P_{\max }^{\mathrm{FAP}}, \forall l \in \mathcal{L} ; \quad \mathrm{P}_{C B S} \leq P_{\max }^{\mathrm{CBS}} .
\end{array}\right.
\end{array}
$$

$$
\begin{aligned}
& \text { P1: } \quad \max _{\boldsymbol{P} \geqq \mathbf{0}, \boldsymbol{Q} \geqq \mathbf{0},\{\mathbf{a}, \mathbf{b}\} \in\{0,1\}} \operatorname{SE}(\boldsymbol{P}, \boldsymbol{Q}, \boldsymbol{a}, \boldsymbol{b}) \\
& \text { s.t. }\left\{\begin{array}{l}
\mathrm{EE}(\boldsymbol{P}, \boldsymbol{Q}, \boldsymbol{a}, \boldsymbol{b}) \geq \epsilon \\
\mathrm{C} 1, \mathrm{C} 2, \mathrm{C} 3, \mathrm{C} 4 .
\end{array}\right.
\end{aligned}
$$

optimal solution to P0. By introducing a lower-bound for the system EE, denoted by $\epsilon$, a single objective formulation of $\mathrm{P} 0$ is obtained as $\mathrm{P} 1$ at the top of current page. Note that $\mathrm{P} 1$ is an EE-SE trade-off optimization problem since each solution to $\mathrm{P} 1$ provides a trade-off between system EE and SE. The following lemma justifies the efficiency of the problem formulation P1,

Lemma 1: P1 provides a weakly Pareto-optimal solution to P0.

Proof: The proof is provided in Appendix A.

It is obvious that different values of $\epsilon$ will lead to different solutions to P1, and Lemma 1 infers that each of these solutions will be weakly Pareto-optimal to P0. Hence, to solve P0 optimally, we need to obtain overall set of the Pareto-optimal solutions by solving P1 for different values of $\epsilon$ and select the solution providing a suitable EE-SE trade-off. However, to do so, the maximum achievable $\mathrm{EE}$ and $\mathrm{SE}$ of the system are required [34]. In what follows, we first obtain the maximum achievable EE and SE of the system, and afterwards, we solve P1 by varying $\epsilon$.

\section{EE-Optimal AND SE-Optimal SOLUTIONS}

To determine the maximum achievable EE and SE of the system by exploiting the considered resource allocation strategy, we formulate the following two optimization problems.

$$
\begin{array}{ccrc}
\text { P2.1 : } & \max _{\boldsymbol{P} \geqq \mathbf{0}, \boldsymbol{Q} \geqq \mathbf{0},\{\mathbf{a}, \mathbf{b}\} \in\{0,1\}} \operatorname{EE}(\boldsymbol{P}, \boldsymbol{Q}, \boldsymbol{a}, \boldsymbol{b}) & \text { s.t. } & \mathrm{C} 1, \mathrm{C} 2, \mathrm{C} 3, \mathrm{C} 4 \\
\text { P2.2: } & \max _{\boldsymbol{P} \geqq \mathbf{0}, \boldsymbol{Q} \geqq \mathbf{0},\{\mathbf{a}, \mathbf{b}\} \in\{0,1\}} \mathrm{SE}(\boldsymbol{P}, \boldsymbol{Q}, \boldsymbol{a}, \boldsymbol{b}) & \text { s.t. } & \mathrm{C} 1, \mathrm{C} 2, \mathrm{C} 3, \mathrm{C} 4 .
\end{array}
$$

According to the well-known Dinkelbach theorem, if $(\boldsymbol{P}, \boldsymbol{Q}, \boldsymbol{a}, \boldsymbol{b})$ is a solution to $\mathrm{P} 2.1$ and $\eta$ is the maximum EE of the system, the following condition must be satisfied.

$$
\max _{\{\boldsymbol{P}, \boldsymbol{Q}, \mathbf{a}, \mathbf{b}\} \mid \mathrm{C} 1, \mathrm{C} 2, \mathrm{C} 3, \mathrm{C} 4}\left(\sum_{j=1}^{K} \sum_{l=1}^{L} \sum_{n=1}^{N} a_{l, j} b_{n, l, j} \mathrm{~T}_{j}^{(l, n)}-\eta\left(\sum_{l \in \mathcal{L}} \mathrm{P}_{l}+\mathrm{P}_{C B S}\right)\right)=0 .
$$


$\mathcal{L}=\sum_{j=1}^{K} \sum_{l=1}^{L} \sum_{n=1}^{N} a_{l, j} b_{n, l, j} \mathcal{T}_{j}^{(l, n)}-\eta\left(\sum_{l \in \mathcal{L}} \mathcal{P}_{l}+\mathcal{P}_{F A P}\right)-\sum_{l \in \mathcal{L}} \lambda_{l}\left(\mathcal{P}_{l}-P_{\text {max }}^{(D)}\right)-\nu\left(\mathcal{P}_{F A P}-P_{\text {max }}^{\mathrm{FAP}}\right)$.

Hence, solving P2.1 is equivalent to finding a resource allocation tuple as such the objective function of the following parameterized optimization problem approaches zero.

$$
\text { P2.3 : } \max _{\substack{\boldsymbol{P} \geq \mathbf{0}, \boldsymbol{Q} \geq \mathbf{0},\{\mathbf{a}, \mathbf{b}\} \in\{0,1\}}} \sum_{j=1}^{K} \sum_{l=1}^{L} \sum_{n=1}^{N} a_{l, j} b_{n, l, j} \mathrm{~T}_{j}^{(l, n)}-\eta\left(\sum_{l \in \mathcal{L}} \mathrm{P}_{l}+\mathrm{P}_{C B S}\right) \quad \text { s.t. } \quad \text { C1,C2,C3,C4. }
$$

However, in P2.3, $\eta$ also depends on the resource allocation variables as depicted from (10). Hence, we first solve P2.3 for a given $\eta$, and then, we update $\eta$ as such (10) is satisfied. Moreover, the solutions to P2.2 and P2.3 converge when $\eta=0$. Thereby, we focus on solving P2.3. Since P2.3 is NP-hard, we propose a modular approach to solve P2.3 with reasonable computational complexity. Particularly, we decompose P2.3 into two sub-problems for performing power control and resource assignment in an iterative manner. The detailed analysis is given as follows.

\section{A. CBS and F-AP power allocation}

Considering that the assignment variables $\{\boldsymbol{a}, \boldsymbol{b}\}$ are given, P2.3 is simplified as

$$
\text { P2.4 : } \max _{\boldsymbol{P} \geqq \mathbf{0}, \boldsymbol{Q} \geq \mathbf{0}} \sum_{j=1}^{K} \sum_{l=1}^{L} \sum_{n=1}^{N} a_{l, j} b_{n, l, j} \mathrm{~T}_{j}^{(l, n)}-\eta\left(\sum_{l \in \mathcal{L}} \mathrm{P}_{l}+\mathrm{P}_{C B S}\right) \quad \text { s.t. C3,C4. }
$$

Although P2.4 is NP-hard, a high quality approximate optimal solution can be obtained by applying Lagrangian relaxation technique [33]. Therefore, to solve P2.4, we formulate a Lagrangian dual-optimization problem and solve it by applying the well-known Karush-Khun-Tucker (KKT) condition. The Lagrangian function corresponding to P2.4 is expressed as (13) at the top of the current page. Here, $\left\{\lambda_{l}\right\}$ and $\nu$ are the Lagrangian multipliers corresponding to C3 and C4 constraints, respectively. Using Lagrangian dual-decomposition, the power allocation problem for the $j$-th device-cluster, $\forall j=1,2, \cdots, K$, is expressed as

$$
\text { P2.5 : } \quad\left\{P_{l, n}^{C, j}, P_{l, e, n}^{p, j}, Q_{n}^{C, j}, Q_{t, n}^{p, j}\right\}=\arg \max _{\mathbb{P} \geqq \mathbf{0}, \mathbb{Q} \geqq \mathbf{0}} \mathcal{L}_{j}
$$

where $\mathcal{L}_{j}$ is defined as (15) at the top of the next page. To apply the KKT condition, P2.5 is decomposed into inner and outer layer problems. The inner and outer layer problems iteratively update the power allocation variables and the Lagrangian multipliers, respectively.

1) Power allocation update: Let the Lagrangian multipliers be given. Capitalizing the IFE based power control method in [39], the near-optimal power allocations of the $j$-th device cluster, 


$$
\mathcal{L}_{j}=\sum_{l=1}^{L} \sum_{n=1}^{N} a_{l, j} b_{l, j, n}\left(\mathcal{T}_{j}^{(l, n)}-\frac{\eta+\lambda_{l}}{\varepsilon}\left(P_{l, n}^{C, j}+\sum_{e \in \mathcal{S}_{j}^{(L)}} P_{l, e, n}^{p, j}\right)-\frac{\eta+\nu}{\varepsilon}\left(Q_{n}^{C, j}+\sum_{t \in \mathcal{S}_{j}^{(H)}} Q_{t, n}^{p, j}\right)\right) .
$$

$$
\begin{aligned}
& P_{l, n}^{C, j}=\frac{\gamma_{l, n}^{C, j} /\left(1+\gamma_{l, n}^{C, j}\right)}{\frac{\eta / \varepsilon+\lambda_{l} / \varepsilon}{1 / \ln 2}+\frac{\left(\widetilde{\gamma}_{n}^{C, j}\right)^{2}\left|h_{\tilde{k}_{j}, n}^{(l)}\right|^{2}}{\left(1+\widetilde{\gamma}_{n}^{C, j}\right) \widehat{Q}_{n}^{C, j}\left|h_{\tilde{k}_{j}, n}^{(0)}\right|^{2}}+\sum_{t \in \mathcal{S}_{j}^{(H)}} \frac{\left(\widetilde{\gamma}_{t, n}^{p, j}\right)^{2}\left|h_{t, n}^{(l)}\right|^{2}}{\left(1+\widetilde{\gamma}_{t, n}^{p, j}\right) \widehat{Q}_{t, n}^{p, j}\left|h_{t, n}^{(0)}\right|^{2}}} \\
& Q_{n}^{C, j}=\frac{\widetilde{\gamma}_{n}^{C, j} /\left(1+\widetilde{\gamma}_{n}^{C, j}\right)}{\frac{\eta / \varepsilon+\nu / \varepsilon}{1 / \ln 2}+\frac{\left(\gamma_{l, n}^{C, j}\right)^{2}\left|h_{k_{j}, n}^{(0)}\right|^{2}}{\left(1+\gamma_{l, n}^{C, j}\right) \widehat{P}_{l, n}^{C, j}\left|h_{k_{j}, n}^{(l)}\right|^{2}}+\sum_{e \in \mathcal{S}_{j}^{(L)}} \frac{\left(\gamma_{l, e, n}^{p, j}\right)^{2}\left|h_{e, n}^{(0)}\right|^{2}}{\left(1+\gamma_{l, e, n}^{p, j}\right) \widehat{P}_{l, e, n}^{p, j}\left|h_{e, n}^{(l)}\right|^{2}}}, \\
& P_{l, e, m}^{p, j}=\frac{\gamma_{l, e, n}^{p, j} /\left(1+\gamma_{l, e, n}^{p, j}\right)}{\frac{\eta+\lambda_{l}}{\varepsilon / \ln 2}+\frac{\left(\gamma_{l, n}^{C, j}\right)^{2}}{\left(1+\gamma_{l, n}^{C, j}\right) \widehat{P}_{l, n}^{C, j}}+\sum_{\begin{array}{c}
e^{\prime} \in \mathcal{S}_{j}^{(L)} \\
e^{\prime} \neq e
\end{array}} \frac{\left(\gamma_{l, e^{\prime}, n}^{\boldsymbol{p}, j}\right)^{2}}{\left(1+\gamma_{l, e^{\prime}, n}^{\boldsymbol{p}, j} \widehat{P}_{l, e^{\prime}, n}^{p, j}\right.}+\frac{\left(\widetilde{\gamma}_{n}^{C, j}\right)^{2}\left|h_{\tilde{k}_{j}, n}^{(l)}\right|^{2}}{\left(1+\widetilde{\gamma}_{n}^{C, j}\right) \widehat{Q}_{n}^{C, j}\left|h_{\tilde{k}_{j}, n}^{(0)}\right|^{2}}+\sum_{t \in \mathcal{S}_{j}^{(H)}} \frac{\left(\widetilde{\gamma}_{t, n}^{p, j}\right)^{2}\left|h_{t, n}^{(l)}\right|^{2}}{\left(1+\widetilde{\gamma}_{t, n}^{\boldsymbol{p}, j}\right) \widehat{Q}_{t, n}^{p, j}\left|h_{t, n}^{(0)}\right|^{2}}},
\end{aligned}
$$

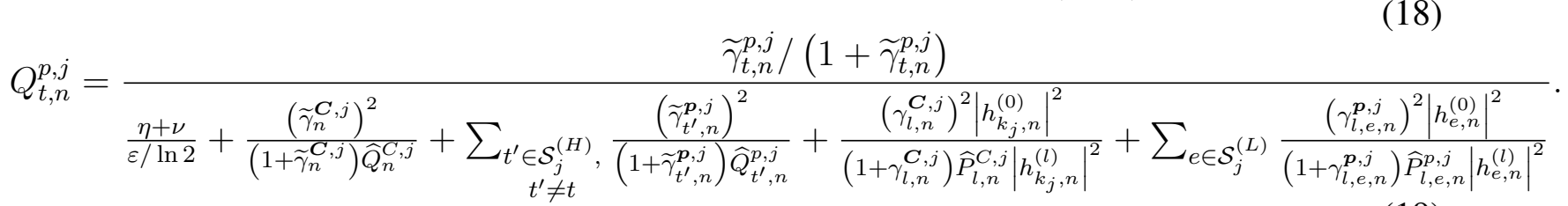

$\forall j=1,2, \cdots, K$, are obtained in the following proposition.

Proposition 1: Let $\left\{\widehat{P}_{l, n}^{C, j}, \widehat{P}_{l, e, n}^{p, j}, \widehat{Q}_{n}^{C, j}, \widehat{Q}_{t, n}^{p, j}\right\}$ be the given power allocations in the $k$-th iteration. A converged power allocation is obtained by updating the power levels at the $(k+1)$-th iteration, $\forall k$, according to (16)-(19) given at the top of the current page. Here, the values of $\gamma_{l, n}^{\boldsymbol{C}, j}, \widetilde{\gamma}_{n}^{\boldsymbol{C}, j}, \gamma_{l, e, n}^{\boldsymbol{p}, j}$, and $\widetilde{\gamma}_{t, n}^{\boldsymbol{p}, j}$ are obtained by plugging $\left\{\widehat{P}_{l, n}^{C, j}, \widehat{P}_{l, e, n}^{p, j}, \widehat{Q}_{n}^{C, j}, \widehat{Q}_{t, n}^{p, j}\right\}$ to (2)-(5).

Proof: The proof is provided in Appendix B.

2) Lagrangian multiplier update: Using (16)-(19), the power consumption of the l-th F-AP and CBS are calculated as $\widehat{\mathrm{P}}_{l}$ and $\widehat{\mathcal{P}}_{C B S}$, respectively. Leveraging the well known sub-gradient method, at the $(k+1)$-th iteration, the Lagrangian multipliers are updated as

$$
\begin{aligned}
& \lambda_{l}[k+1]=\max \left[\lambda_{l}[k]+\zeta_{1}(k)\left(\widehat{\mathrm{P}}_{l}-P_{\max }^{\mathrm{FAP}}\right), 0\right], \forall l \in \mathcal{L} \\
& \nu[k+1]=\max \left[\nu[k]+\zeta_{2}(k)\left(\widehat{\mathrm{P}}_{C B S}-P_{\max }^{\mathrm{CBS}}\right), 0\right]
\end{aligned}
$$

where $\left\{\zeta_{1}(k), \zeta_{2}(k)\right\}$ are the step sizes at the $k$-th iteration, and for convergence, the step sizes are selected as such $\sum_{k=1}^{\infty} \zeta_{i}(k)=\infty$ and $\sum_{k=1}^{\infty} \zeta_{i}^{2}(k)<\infty, i=1,2$ are satisfied. 
3) Algorithm development: We propose Algorithm 1 to obtain power allocations in the $j$-th device-cluster. For a given device-cluster, Algorithm 1 requires $\mathcal{O}(L N)$ computational complexity to obtain a converged solution to P2.4. To demonstrate efficiency of Algorithm 1, we introduce the following proposition.

Proposition 2: Algorithm 1 converges to a stable and near-optimal solution to P2.4.

Proof: The proof is provided in Appendix $C$

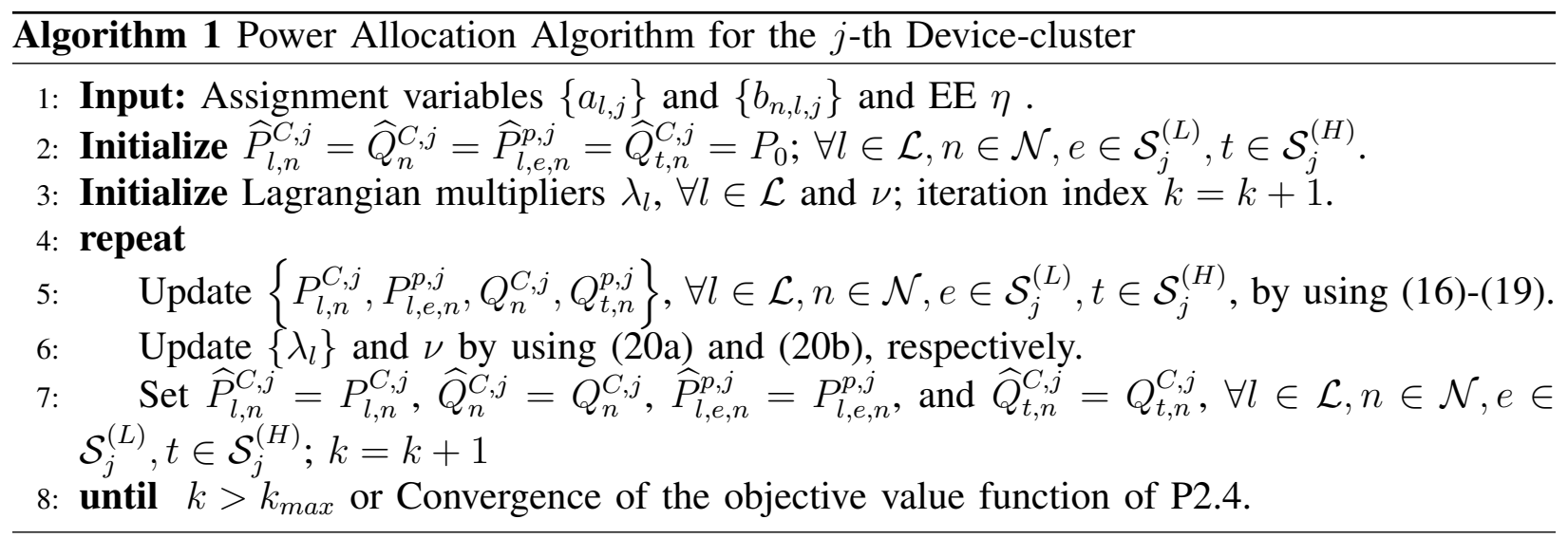

\section{B. 3D-Resource Matching}

1) Algorithm development: For a given set of power allocation variables, P2.3 is written as

$$
\text { P2.6 : } \max _{\{\mathbf{a}, \mathbf{b}\} \in\{0,1\}} \sum_{j=1}^{K} \sum_{l=1}^{L} \sum_{n=1}^{N} a_{l, j} b_{n, l, j} \widehat{U}_{l, n}^{(j)} \quad \text { s.t. } \quad \text { C1, C2 }
$$

where $\widehat{U}_{l, n}^{(j)}$ is a function of the power allocations obtained from Algorithm 1, and it is given by

$$
\widehat{U}_{l, n}^{(j)}=\mathrm{T}_{j}^{(l, n)}\left(P_{l, n}^{C, j}, P_{l, e, n}^{p, j}, Q_{n}^{C, j}, Q_{t, n}^{p, j}\right)-\frac{\eta}{\varepsilon}\left(P_{l, n}^{C, j}+\sum_{e \in \mathcal{S}_{j}^{(L)}} P_{l, e, n}^{p, j}+Q_{n}^{C, j}+\sum_{t \in \mathcal{S}_{j}^{(H)}} Q_{t, n}^{p, j}\right) .
$$

To solve P2.6, we model the problem of assigning F-APs and RRBs among the device-clusters as a 3D-resource matching problem. The rules of matching and preference-orders of the deviceclusters, F-APs, and RRBs are defined as follows.

Definition 1: A 3D-matching is defined as a function $\Phi(\cdot, \cdot)$ that maps elements of the following sets, $\mathcal{S} \times \mathcal{L}, \mathcal{S} \times \mathcal{N}$, and $\mathcal{L} \times \mathcal{N}$, into the elements of following sets $\mathcal{N}$, $\mathcal{L}$, and $\mathcal{S}$, respectively. For all $\{j, l, n\} \in\{\mathcal{S}, \mathcal{L}, \mathcal{N}\}$, such a mapping satisfies the following conditions.

1) $\boldsymbol{\Phi}(j, n) \in \mathcal{L}$ and $\boldsymbol{\Phi}(j, n)=\boldsymbol{\Phi}(j, \tilde{n}), \forall n, \tilde{n} \in \mathcal{N}, n \neq \tilde{n}$.

2) $\boldsymbol{\Phi}(l, n) \in \mathcal{S}, \boldsymbol{\Phi}(l, n) \neq \boldsymbol{\Phi}(\tilde{l}, \tilde{n}), \forall n \neq \tilde{n}, n, \tilde{n} \in \mathcal{N}$ and $\forall l \neq \tilde{l}, l, \tilde{l} \in \mathcal{L}$.

3) $|\boldsymbol{\Phi}(l, n)| \leq 1$ and $\left|\cup_{n \in \mathcal{N}_{s c}} \boldsymbol{\Phi}(l, n)\right| \leq N_{R}$. 
4) $\boldsymbol{\Phi}(j, l) \subset \mathcal{N}, \boldsymbol{\Phi}(j, l) \cap \boldsymbol{\Phi}(\tilde{j}, l)=\varnothing, \boldsymbol{\Phi}(j, l) \cap \boldsymbol{\Phi}(\tilde{j}, \tilde{l})=\varnothing, \forall j, \tilde{j} \in \mathcal{S}, l, \tilde{l} \in \mathcal{L}, j \neq \tilde{j}, l \neq \tilde{l}$.

5) $\boldsymbol{\Phi}(j, l)=\varnothing \Longleftrightarrow \nexists n, \boldsymbol{\Phi}(j, n)=l$

6) $\boldsymbol{\Phi}(j, n)=l \Longleftrightarrow \boldsymbol{\Phi}(l, n)=j \Longleftrightarrow \boldsymbol{\Phi}(j, l)=n$.

Condition 1) implies that if the $n$-th and $\tilde{n}$-th RRBs are matched with the $j$-th device-cluster, then both the $(j, n)$ and $(j, \tilde{n})$ pairs will be matched with the same F-AP. Condition 2) implies that if the $n$-th and $\tilde{n}$-th RRBs are matched with the $l$-th and $\tilde{l}$-th F-APs, respectively, then the $(l, n)$ and $(\tilde{l}, \tilde{n})$ pairs must be matched with different device-clusters. Condition 3) implies that the $(l, n)$ pair can be matched with maximum one device-cluster, and the total number of matched device-clusters with the l-th F-AP over all the RRBs can not exceed $N_{R}$. Condition 4) implies that two different device-clusters will always be matched with orthogonal sets of RRBs. Finally, Conditions 5) and 6) imply that the j-th device-cluster, the l-th F-AP, and the $n$-th RRB will be matched if and only if any of these two elements are matched with the third element.

Definition 2: To maximize the objective function value of P2.6, the $j$-th device-cluster prefers the $(l, n)$ pair over the $\left(l^{\prime}, n^{\prime}\right)$ pair if the following condition is satisfied

$$
(l, n) \succ_{j}\left(l^{\prime}, n^{\prime}\right) \Longleftrightarrow \widehat{U}_{l, n}^{(j)}>\widehat{U}_{l^{\prime}, n^{\prime}}^{(j)} \quad \forall l, l^{\prime} \in \mathcal{L}, \forall n, n^{\prime} \in \mathcal{N}
$$

The l-th F-AP prefers the $(j, n)$ pair over the $\left(j^{\prime}, n^{\prime}\right)$ pair if the following condition is satisfied

$$
(j, n) \succ_{l}\left(j^{\prime}, n^{\prime}\right) \Longleftrightarrow \widehat{U}_{l, n}^{(j)}>\widehat{U}_{l, n^{\prime}}^{\left(j^{\prime}\right)} \quad \forall j, j^{\prime} \in \mathcal{S}, \forall n, n^{\prime} \in \mathcal{N}
$$

The n-the RRB prefers the $(j, l)$ pair over the $\left(j^{\prime}, l^{\prime}\right)$ pair if the following condition is satisfied

$$
(j, l) \succ_{n}\left(j^{\prime}, l^{\prime}\right) \Longleftrightarrow \widehat{U}_{l, n}^{(j)}>\widehat{U}_{l^{\prime}, n}^{\left(j^{\prime}\right)} \quad \forall j, j^{\prime} \in \mathcal{S}, \forall l, l^{\prime} \in \mathcal{L}
$$

In this matching problem, as the players are matched with their preferred pairs according to (23)-(25), either the system SE is increased or the power consumption of the system is reduced. However, for this matching problem, finding a stable 3D-matching is NP-hard and computationally intractable [35]. Consequently, we develop a heuristic algorithm to obtain matching among the device-clusters, F-APs, and RRBs. The overall matching has the following three phases.

F-AP matching (FM) phase: In FM phase, the device-clusters are matched with suitable F-APs as such a single device-cluster is matched with only one F-AP and any F-AP is matched with maximum $N_{R}$ device-clusters. In this phase, two different sets, namely, $\mathbf{U M}_{D}$ and $\mathbf{U M}_{R}$ are maintained for the unmatched device-clusters and the F-APs capable of accepting deviceclusters, respectively. At each iteration of the FM phase, an eligibility score, $\mathrm{D}_{j}$, is calculated for $\forall j \in \mathbf{U M}_{D}$, and it provides the eligibility of the unmatched device-clusters to be matched 
with their most preferred F-AP in the $\mathrm{UM}_{R}$ set. The unmatched device-cluster having maximum eligibility score is matched with its most preferred F-AP in the $\mathbf{U M}_{R}$ set based on (23), and such a device-cluster is removed from the $\mathbf{U M}_{D}$ set. The $\mathbf{U M}_{R}$ set is updated as well. The FM phase is continued until all the device-clusters are matched with certain F-APs.

RRB matching (RM) phase: In RM phase, the remaining RRBs, which are not matched with any device-cluster, are allocated among the device-clusters. The RM phase is a two-sided many-to-one matching problem where the matched device-cluster and F-APs in the FM phase constitute one side, and the unmatched RRBs constitute other side. Each unmatched RRB is matched with its most preferred device-cluster and F-AP pair according to (25). The RM phase is continued until all the RRBs are matched.

RRB swapping (RS) phase: In RS phase, a set of RRBs between any two device-clusters are swapped given the overall utility of the both device-clusters is improved. To explain the RS operation, we assume that $\boldsymbol{\Phi}(j, l)=n$ and $\boldsymbol{\Phi}(\tilde{j}, \tilde{l})=\tilde{n}$. If $\widehat{U}_{l, \tilde{n}}^{(j)}+\widehat{U}_{\tilde{l}, n}^{(\tilde{j})}>\widehat{U}_{l, n}^{(j)}+\widehat{U}_{\tilde{l}, \tilde{n}}^{(\tilde{j})}$ is satisfied, the $(n, \tilde{n})$-th RS are swapped between the $j$-th and $\tilde{j}$-th device-clusters. The RS phase is continued until there is no swappable device-cluster pair in the system.

The overall steps of the proposed 3D-resource matching are summarized in Algorithm 2. In Algorithm 2, $L_{j}^{*}$ denotes the assigned F-AP to the $j$-th device-cluster, $\mathcal{N}_{j}^{*}$ denotes set of the assigned RRBs to the $j$-th device-cluster, $K_{l}$ is the number of device-clusters associated with the $l$-th F-AP, and $\mathrm{UM}_{N}$ is the set of the unmatched RRBs.

2) Property Analysis of Algorithm 2: Although the proposed Algorithm 2 is heuristic, it provides a single-sided Pareto-efficient matching for the device-clusters. To this end, we first introduce the following definitions.

Definition 3: Two device-clusters will be Pareto-improvement pair if by partially or completely exchanging their matched resources with each other, their overall utility is strictly improved.

Definition 4: The matching obtained by Algorithm 2 will be Pareto-efficient for the deviceclusters if it does not contain any Pareto-improvement pair.

Proposition 3: The final matching obtained by Algorithm 2, $\Phi^{*}$, is Pareto-efficient for the device-clusters.

Proof: The proof is provided in Appendix D.

Consequently, despite being heuristic, Algorithm 2 is efficient. We obtain solution to P2.6 from $\boldsymbol{\Phi}^{*}$ as follows. Particularly, $\forall j \in \mathcal{S}, a_{l, j}=1$ and $b_{n, l, j}=1$ if $\boldsymbol{\Phi}^{*}(j, n)=l, \boldsymbol{\Phi}^{*}(l, n)=$ $j, \Phi^{*}(j, l)=n$, and $a_{l, j}=0$ and $b_{n, l, j}=0$, otherwise. The following proposition establishes the near-optimality of the derived solutions. 
Proposition 4: For the given power allocations, Algorithm 2 provides a near-optimal solution to P2.6.

Proof: The proof is provided in Appendix E.

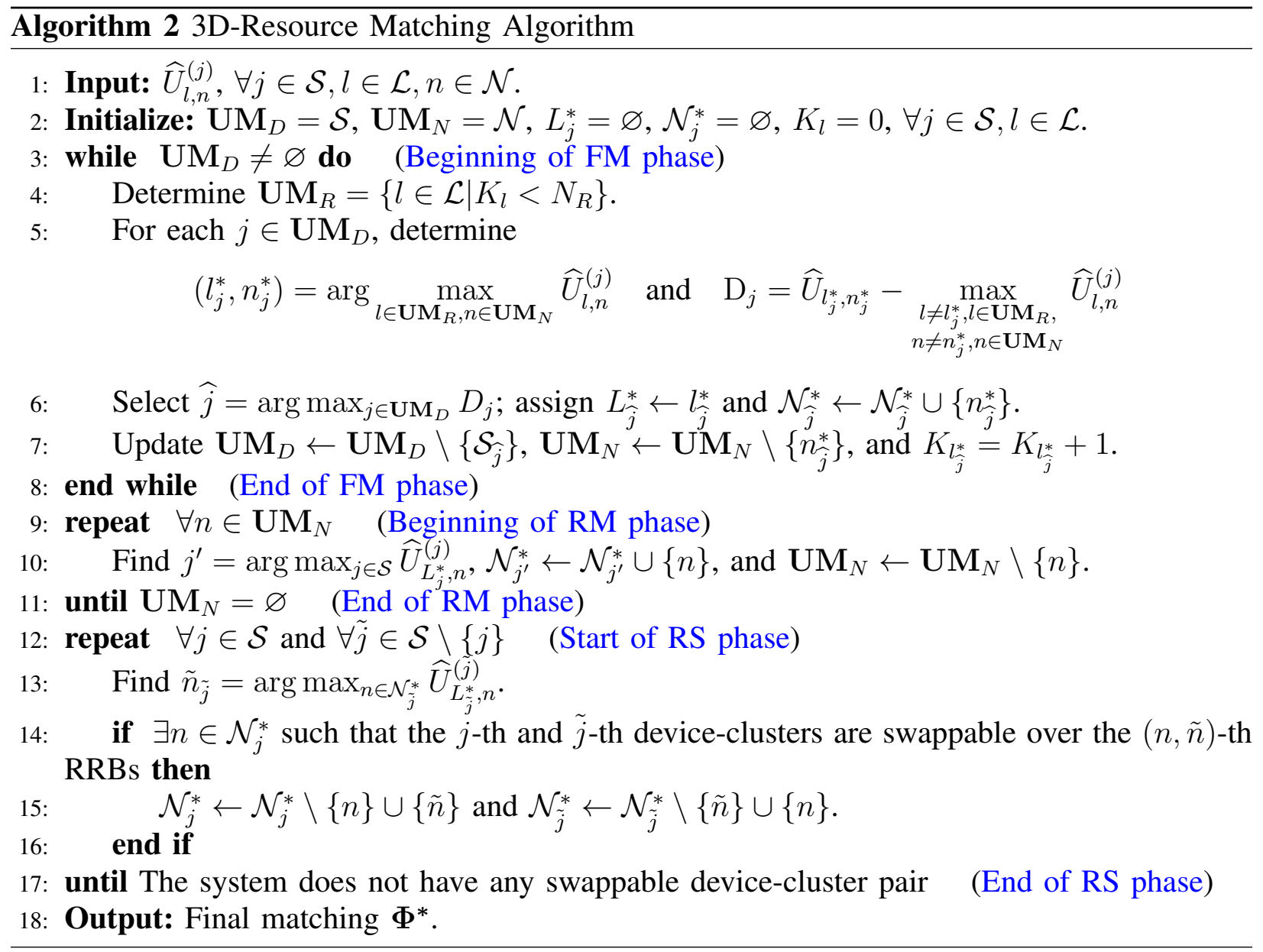

The computational complexity of Algorithm 2 is explained as follows. Particularly, in each iteration of the FM phase in Algorithm 2, a single device-cluster is matched with an F-AP and RRB pair. In each iteration of the FM phase, the metric $\left\{D_{j}\right\}$ is calculated for all the unmatched device-clusters, and the set $\mathbf{U} \mathbf{M}_{R}$ is computed for the F-APs. Therefore, the required computation of the FM phase is $\sum_{j=1}^{|\mathcal{S}|} j+L=\frac{|S|(|S|+1)}{2}+|S| L$. For each unmatched RRB in the RM phase, $\mathcal{O}(|S|)$ operation is needed to execute the Step (10) of the Algorithm 2. Since the total number of unmatched RRBs in the RM phase is $N-|S|$, the total number of required computations is $(N-|S|)|S|$. Finally, for performing the RS phase, each device-cluster has to visit the rest of the $|S|-1$ device-clusters. Therefore, in the worst-case, total required computations to execute the Steps (14) and (15) in Algorithm 2 is $|S|(|S|-1) N$. By combining the required computations of the FM, RM, and RS phases and considering the fact that $|S|=K$, the worst-case computational complexity of Algorithm 2 is obtained as $\mathcal{O}\left(|S|^{2} N+|S| L\right)=\mathcal{O}\left(K^{2} N+K L\right)$. 


\section{Algorithms for Maximizing EE and SE}

Capitalizing the IFE based power control and 3D-resource matching, we develop Algorithm 3 and Algorithm 4 to determine maximum achievable EE and SE of the system, respectively. Both Algorithm 3 and Algorithm 4 iteratively update solutions to P2.4 and P2.6 by adopting a block-alternating accent method. Since both P2.4 and P2.6 are near-optimally solved, Algorithm 3 and Algorithm 4 must converge as confirmed by the following proposition.

Proposition 5: Algorithm 3 and Algorithm 4 converge to near-optimal system EE and SE, respectively.

Proof: The proof is provided in Appendix $F$.

The computational complexity of Algorithm 3 and Algorithm 4 is explained as follows. Let the convergence of Algorithm 3 and Algorithm 4 requires $\Delta_{\max }^{(1)}$ iterations. Using the reported computational complexity of Algorithms 1 and 2, the worst-case computational complexity of Algorithm 3 and Algorithm 4 is obtained as $\mathcal{O}\left(\Delta_{\max }^{(1)}\left(K L N+K^{2} N+K L\right)\right) \approx$ $\mathcal{O}\left(\Delta_{\max }^{(1)}\left(K L N+K^{2} N\right)\right)$.
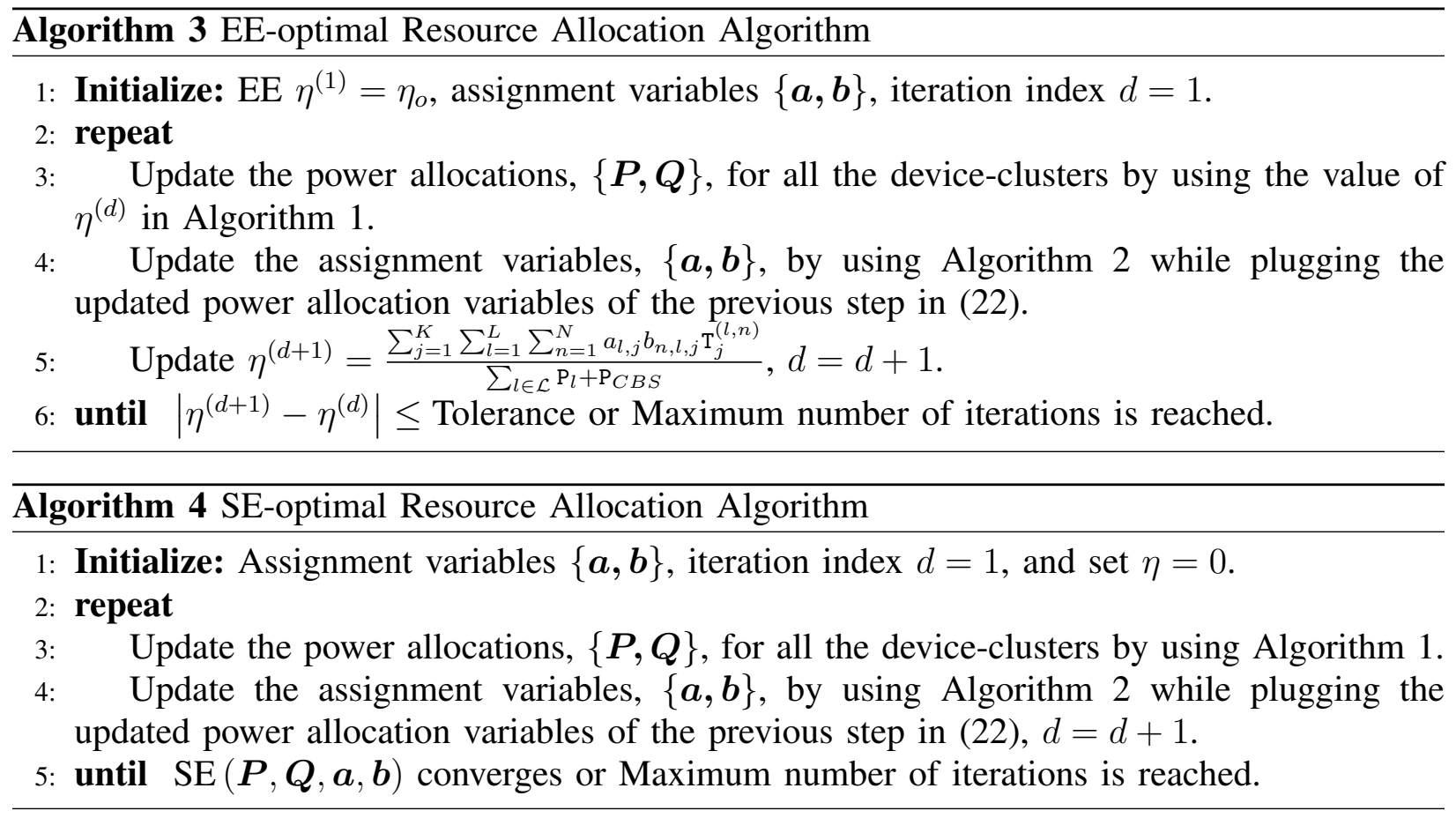

\section{EE-SE Trade-OFF Optimization AND Proposed Algorithms}

\section{A. Near-optimal Algorithm Design}

We obtain the Pareto-front of P0 by solving P1. P1 can be solved by applying a Lagrangian optimization technique. Let $\mu$ be the Lagrangian multiplier correspond to C5 constraint in P1. 
The partial Lagrangian of P1 is written as

$$
\mathcal{L}^{(2)}=(1+\mu) \sum_{j=1}^{K} \sum_{l=1}^{L} \sum_{n=1}^{N} a_{l, j} b_{n, l, j} \mathrm{~T}_{j}^{(l, n)}-\mu \epsilon\left(\sum_{l \in \mathcal{L}} \mathrm{P}_{l}+\mathrm{P}_{C B S}\right)
$$

For a given value of $\epsilon$, a local optimal solution to P1 can be obtained as

$$
\text { P3.1: }\left\{\boldsymbol{P}^{*}(\epsilon), \boldsymbol{Q}^{*}(\epsilon), \boldsymbol{a}^{*}(\epsilon), \boldsymbol{b}^{*}(\epsilon)\right\}=\arg \min _{\mu \geq 0} \max _{\substack{\mathbb{P} \geq \mathbf{0}, \mathbb{Q} \geq \mathbf{0},\{\mathbf{a}, \mathbf{b}\} \in\{0,1\}}} \mathcal{L}^{(2)} \quad \text { s.t. } \quad \text { C1,C2,C3,C4. }
$$

Note that, P3.1 and P2.3 have a similar structure except some scalar multipliers. Hence, for the given values of $\mu$ and $\epsilon$, Algorithm 1 and Algorithm 2 are applied to obtain $\left\{\boldsymbol{P}^{*}(\epsilon), \boldsymbol{Q}^{*}(\epsilon)\right\}$ and $\left\{\boldsymbol{a}^{*}(\epsilon), \boldsymbol{b}^{*}(\epsilon)\right\}$, respectively. To avoid redundancy, the detailed analysis is omitted. Meanwhile, at the optimality of P3.1, $\mu^{*}>0$ will be satisfied; otherwise, P3.1 will converge to an SE maximization problem. According to the complementary slackness condition, an optimal value of $\mu^{*}$ must be obtained as such the C5 constraint becomes active. Since P3.1 provides a (local) optimal solution to P1, such a solution is also weakly Pareto optimal to P0. The overall set of the (weakly) Pareto optimal solutions to P0 is defined as the Pareto-front of P0. To obtain the Pareto-front of $\mathrm{P} 0$, we introduce the following proposition.

Proposition 6: Let $\left\{\boldsymbol{P}_{S E}, \boldsymbol{Q}_{S E}, \boldsymbol{a}_{S E}, \boldsymbol{b}_{S E}\right\}$ and $\left\{\boldsymbol{P}_{E E}, \boldsymbol{Q}_{E E}, \boldsymbol{a}_{E E}, \boldsymbol{b}_{E E}\right\}$ are the SE-optimal and EE-optimal resource allocation variables. The near-optimal Pareto-front of PO can be achieved by solving P3.1 while varying $\epsilon$ in the range $\boldsymbol{E} \boldsymbol{E}\left(\boldsymbol{P}_{S E}, \boldsymbol{Q}_{S E}, \boldsymbol{a}_{S E}, \boldsymbol{b}_{S E}\right) \leq \epsilon \leq$ $\boldsymbol{E} \boldsymbol{E}\left(\boldsymbol{P}_{E E}, \boldsymbol{Q}_{E E}, \boldsymbol{a}_{E E}, \boldsymbol{b}_{E E}\right)$.

Proof: The proof is provided in Appendix $G$.

Based on Proposition 6, we propose an I-RSRM algorithm. The overall steps of I-RSRM algorithm are summarized in Algorithm 5. The total number of the solutions obtained by IRSRM algorithm is $N_{F}$, and each of theses solutions provides a certain trade-off between $\mathrm{EE}$ and SE of the system. However, since P0 is a non-convex and NP-hard multi-objective optimization problem, the global optimality of the Pareto-front obtained by I-RSRM algorithm is not guaranteed. Nevertheless, by selecting a small step size $\Delta$ and increasing $N_{F}$, I-RSRM can obtain a good approximation of the optimal Pareto-front. Accordingly, I-RSRM provides a near-optimal Pareto-front for the multi-objective resource optimization problem, P0.

\section{B. Low-Complexity Algorithm Design}

Despite near-optimality, I-RSRM algorithm requires significant large number of iterations, and number of iterations increases with the size of network. Consequently, the convergence rate of I-RSRM algorithm may not affordable in large scale network. To reduce the number of iterations, 
we propose D-RSRM algorithm. The overall steps of D-RSRM are summarized in Algorithm 6. Unlike I-RSRM, D-RSRM decouples the power allocations and resource assignment. Particularly, D-RSRM initially determines the assignment of the F-APs and RRBs among the device-clusters by considering a uniform power allocation, and keeps such assignments fixed for the remaining steps. Essentially, the total number of iterations is substantially reduced in D-RSRM. Similar to I-RSRM, D-RSRM provides a set of resource allocation variables where each solution has a certain EE-SE trade-off. However, the Pareto-front obtained by D-RSRM is sub-optimal.

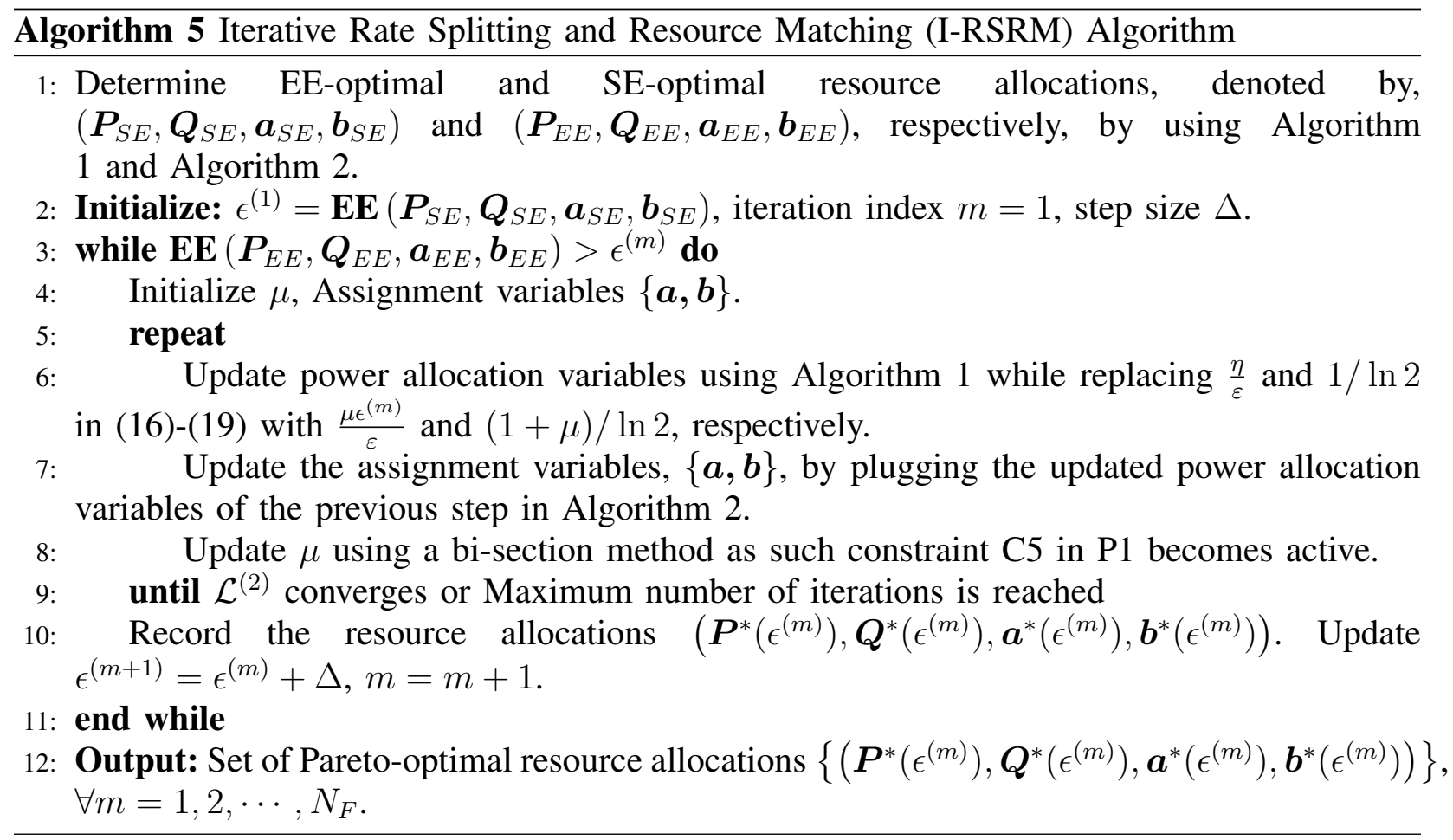

\section{Operating EE-SE pair selection Algorithm}

In this section, we explain the procedure of selecting a suitable operating EE-SE pair from the Pareto-front obtained by the proposed I-RSRM and D-RSRM algorithms. Note that the decision of the system to trade-off certain portion of its optimal EE (or optimal SE) can be perceived as a cost paid by the system to achieve certain profit in terms of SE (or EE) improvement. Assume that the system allows maximum $\alpha$ percentage loss of its optimal EE or SE. Thus, the system can either trade-off maximum $\alpha$ percentage of the optimal EE or the optimal SE. Here, $\alpha$ is a system defined EE-SE trade-off control parameter. We need to decide whether it is beneficial to trade-off $\alpha$ percentage of optimal EE (resp. optimal SE) for having a gain in the achievable SE (resp. achievable EE) of the system. To this end, we denote $\boldsymbol{E} \boldsymbol{E}^{* *}$ and $\boldsymbol{S} \boldsymbol{E}^{* *}$ by the achievable EE and SE given the system is willing to trade-off $\alpha$ percentage of optimal EE and SE, respectively. 


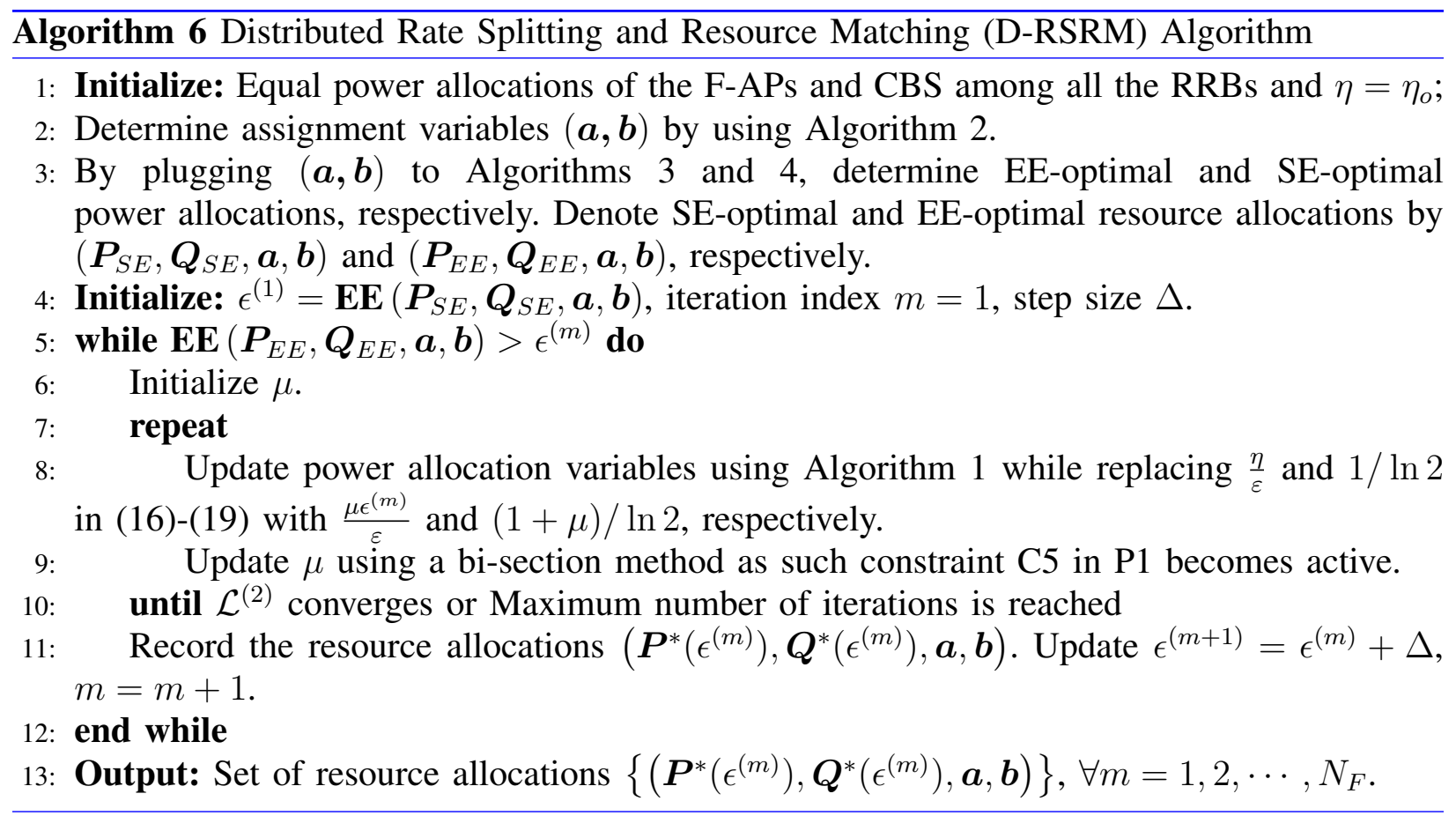

Let, $\boldsymbol{E} \boldsymbol{E}^{*}$ and $\boldsymbol{S} \boldsymbol{E}^{*}$ be the maximum EE and SE in the available Pareto-front, respectively. To determine $\boldsymbol{E} \boldsymbol{E}^{* *}$ and $\boldsymbol{S} \boldsymbol{E}^{* *}$, we formulate the following two optimization problems.

$$
\begin{aligned}
& \boldsymbol{E} \boldsymbol{E}^{* *}=\underset{\left\{\left(\boldsymbol{P}^{*}\left(\epsilon^{(m)}\right), \boldsymbol{Q}^{*}\left(\epsilon^{(m)}\right), \boldsymbol{a}^{*}\left(\epsilon^{(m)}\right), \boldsymbol{b}^{*}\left(\epsilon^{(m)}\right)\right)\right\}}{\max } \boldsymbol{E} \boldsymbol{E}\left(\boldsymbol{P}^{*}\left(\epsilon^{(m)}\right), \boldsymbol{Q}^{*}\left(\epsilon^{(m)}\right), \boldsymbol{a}^{*}\left(\epsilon^{(m)}\right), \boldsymbol{b}^{*}\left(\epsilon^{(m)}\right)\right) \\
& \text { s.t. } \quad \boldsymbol{S E}\left(\boldsymbol{P}^{*}\left(\epsilon^{(m)}\right), \boldsymbol{Q}^{*}\left(\epsilon^{(m)}\right), \boldsymbol{a}^{*}\left(\epsilon^{(m)}\right), \boldsymbol{b}^{*}\left(\epsilon^{(m)}\right)\right) \geq\left(1-\frac{\alpha}{100}\right) \boldsymbol{S} \boldsymbol{E}^{*} . \\
& \boldsymbol{S} \boldsymbol{E}^{* *}=\underset{\left\{\left(\boldsymbol{P}^{*}\left(\epsilon^{(m)}\right), \boldsymbol{Q}^{*}\left(\epsilon^{(m)}\right), \boldsymbol{a}^{*}\left(\epsilon^{(m)}\right), \boldsymbol{b}^{*}\left(\epsilon^{(m)}\right)\right)\right\}}{\boldsymbol{S E}\left(\boldsymbol{P}^{*}\left(\epsilon^{(m)}\right), \boldsymbol{Q}^{*}\left(\epsilon^{(m)}\right), \boldsymbol{a}^{*}\left(\epsilon^{(m)}\right), \boldsymbol{b}^{*}\left(\epsilon^{(m)}\right)\right)} \\
& \text { s.t. } \quad \boldsymbol{E} \boldsymbol{E}\left(\boldsymbol{P}^{*}\left(\epsilon^{(m)}\right), \boldsymbol{Q}^{*}\left(\epsilon^{(m)}\right), \boldsymbol{a}^{*}\left(\epsilon^{(m)}\right), \boldsymbol{b}^{*}\left(\epsilon^{(m)}\right)\right) \geq\left(1-\frac{\alpha}{100}\right) \boldsymbol{E} \boldsymbol{E}^{*} .
\end{aligned}
$$

In both (28) and (29), the resource allocation variables are taken from the output of the proposed I-RSRM (D-RSRM) algorithm. Hence, both (28) and (29) are solved by performing simple search over the EE and SE pairs in the obtained Pareto-front. Thereby, we obtain two possible operating EE-SE pairs, such as, (i) $\left(\boldsymbol{E} \boldsymbol{E}^{* *},\left(1-\frac{\alpha}{100}\right) \boldsymbol{S} \boldsymbol{E}^{*}\right)$ obtained by trading-off $\alpha$ percentage of optimal SE and (ii) $\left(\left(1-\frac{\alpha}{100}\right) \boldsymbol{E} \boldsymbol{E}^{*}, \boldsymbol{S} \boldsymbol{E}^{* *}\right)$ obtained by trading-off $\alpha$ percentage of optimal EE. For the ease of description, we denote $\left(\boldsymbol{E} \boldsymbol{E}^{* *},\left(1-\frac{\alpha}{100}\right) \boldsymbol{S} \boldsymbol{E}^{*}\right)$ and $\left(\left(1-\frac{\alpha}{100}\right) \boldsymbol{E} \boldsymbol{E}^{*}, \boldsymbol{S} \boldsymbol{E}^{* *}\right)$ as EE-SE pair-A and EE-SE pair-B, respectively. Our target is to select either EE-SE pair-A or EE-SE pair-B as such the benefit of trade-off (B.O.T) is maximized. To calculate the B.O.T metric, we require the following information: (1) The net (relative) EE gain of EE-SE pair-A for trading-off 
$\alpha$ percentage of optimal SE, and the net (relative) SE gain of EE-SE pair-B for trading-off $\alpha$ percentage of optimal EE; (2) the relative gain (or loss) of EE for selecting EE-SE pair-A (resp. EE-SE pair-B) instead of EE-SE pair-B (resp. EE-SE pair-A), and (3) the relative gain (or loss) of SE for selecting EE-SE pair-A (resp. EE-SE pair-B) instead of EE-SE pair-B (resp. EE-SE pair-A). By linearly combining the aforementioned gains, we obtain the B.O.T metrics for both EE-SE pair-A and EE-SE pair-B. Then, we select the EE-SE pair having the maximum B.O.T as the operating EE-SE pair. The B.O.T metrics for EE-SE pair-A and EE-SE pair B are calculated as, respectively,

$$
\begin{aligned}
\mathcal{B O} \mathcal{T}_{1} & =w_{1}\left(\chi_{E E}-\alpha\right)+w_{2} \frac{\boldsymbol{E} \boldsymbol{E}^{* *}-\left(1-\frac{\alpha}{100}\right) \boldsymbol{E} \boldsymbol{E}^{*}}{\left(1-\frac{\alpha}{100}\right) \boldsymbol{E} \boldsymbol{E}^{*}}+w_{3} \frac{\left(1-\frac{\alpha}{100}\right) \boldsymbol{S} \boldsymbol{E}^{*}-\boldsymbol{S} \boldsymbol{E}^{* *}}{\boldsymbol{S} \boldsymbol{E}^{* *}} \\
\mathcal{B O} \mathcal{T}_{2} & =w_{1}\left(\chi_{S E}-\alpha\right)+w_{2} \frac{\left(1-\frac{\alpha}{100}\right) \boldsymbol{E} \boldsymbol{E}^{*}-\boldsymbol{E} \boldsymbol{E}^{* *}}{\boldsymbol{E} \boldsymbol{E}^{* *}}+w_{3} \frac{\boldsymbol{S \boldsymbol { E } ^ { * * }}-\left(1-\frac{\alpha}{100}\right) \boldsymbol{S} \boldsymbol{E}^{*}}{\left(1-\frac{\alpha}{100}\right) \boldsymbol{S} \boldsymbol{E}^{*}} .
\end{aligned}
$$

In (30a) and (30b), $\left\{w_{1}, w_{2}, w_{3}\right\}$ is a set of network determined weight-factors. In (30a) and (30b), $\chi_{E E}$ and $\chi_{S E}$ are defined as, respectively,

$$
\begin{aligned}
& \chi_{E E}=\frac{\boldsymbol{E} \boldsymbol{E}^{* *}-\widehat{E E}_{\boldsymbol{S E}=\boldsymbol{S} \boldsymbol{E}^{*}}}{\widehat{E E}_{\boldsymbol{S E}=\boldsymbol{S} \boldsymbol{E}^{*}}} \times 100 \\
& \chi_{S E}=\frac{\boldsymbol{S} \boldsymbol{E}^{* *}-\widehat{S E}_{\boldsymbol{E} \boldsymbol{E}=\boldsymbol{E} \boldsymbol{E}^{*}}}{\widehat{S E}_{\boldsymbol{E} \boldsymbol{E}=\boldsymbol{E} \boldsymbol{E}^{*}}} \times 100
\end{aligned}
$$

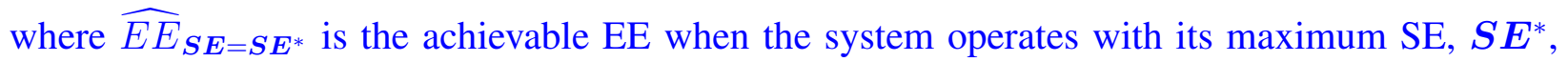
and $\widehat{S E}_{\boldsymbol{E} \boldsymbol{E}=\boldsymbol{E} \boldsymbol{E}^{*}}$ is the achievable SE when the system operates with its maximum EE, $\boldsymbol{E} \boldsymbol{E}^{*}$. Here, $\chi_{E E}$ provides the percentage gain of EE when the system allows maximum $\alpha$ percentage loss of its optimal SE, and $\chi_{S E}$ provides the percentage gain of SE when the system allows maximum $\alpha$ percentage loss of its optimal EE. Obviously, if $\mathcal{B O} \mathcal{T}_{1}>\mathcal{B O}_{2}$ holds, it is more beneficial to trade-off $\alpha$ percentage of the optimal SE and the operating EE-SE pair of the system is selected as $\left(\boldsymbol{E} \boldsymbol{E}^{* *},\left(1-\frac{\alpha}{100}\right) \boldsymbol{S} \boldsymbol{E}^{*}\right)$. Conversely, if $\mathcal{B O} \mathcal{T}_{2}>\mathcal{B O} \mathcal{T}_{1}$ holds, it is more beneficial to trade-off $\alpha$ percentage of the optimal EE and the operating EE-SE pair of the system is selected as $\left(\left(1-\frac{\alpha}{100}\right) \boldsymbol{E} \boldsymbol{E}^{*}, \boldsymbol{S} \boldsymbol{E}^{* *}\right)$. The overall steps of selecting a suitable EE-SE pair and the corresponding resource allocation variables are summarized in Algorithm 7. Note that the optimality of Algorithm 7's output depends on the optimality of the available Paretofront. Accordingly, by increasing the number of solutions of I-RSRM and D-RSRM algorithms, the near-optimality of both the obtained Pareto-front and Algorithm 7's output can be improved. 


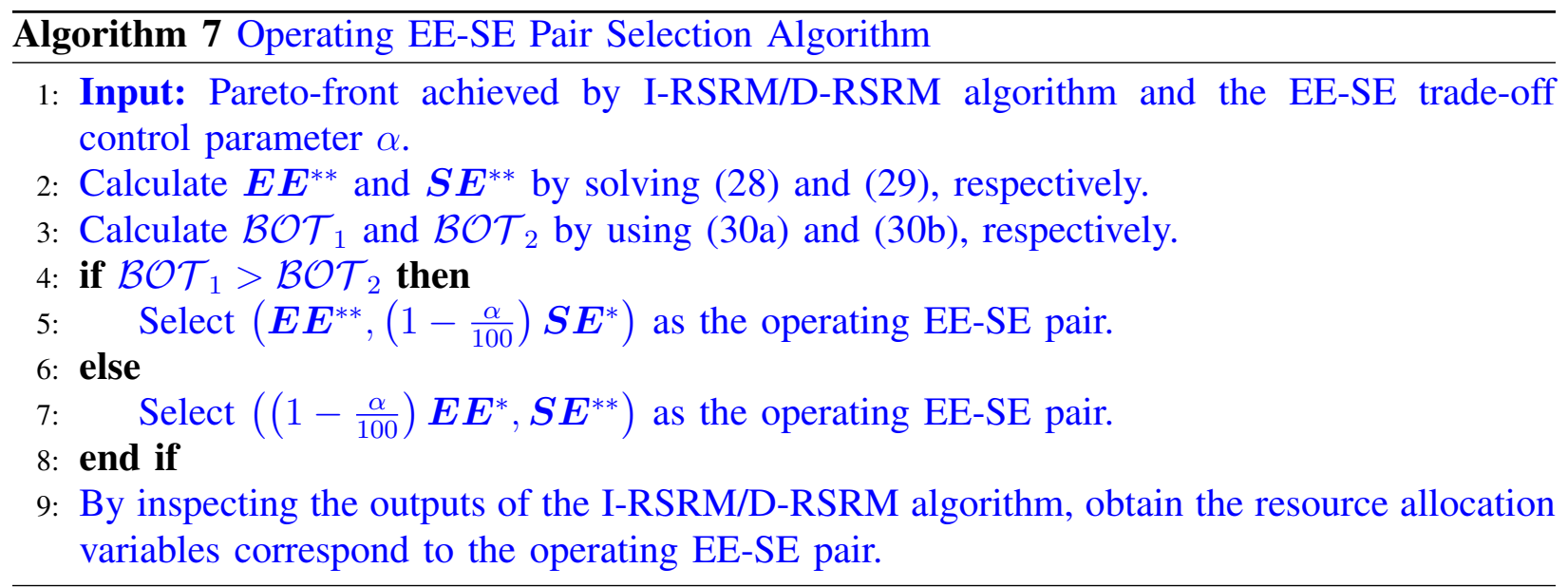

\section{Implementation and Computational Complexity Analysis}

Implementation: Leveraging the reliable fronthaul links between CBS and F-APs and the computation resources of the CBS, both I-RSRM and D-RSRM algorithms are executed at the CBS. In each TS, CBS first collects the global CSI of the network. Then, CBS obtains the Pareto-front of system EE and SE by executing the proposed I-RSRM/D-RSRM algorithm. Subsequently, by executing Algorithm 7, CBS determines the operating EE-SE pair and the corresponding resource allocations. Finally, CBS coordinates the resource allocation decisions with the F-APs via the fronthaul links. Note that the fronthaul links are mainly used for coordination/scheduling purpose, and hence, the burden over fronthaul is relieved. In this regard, the proposed implementation is in agreement with the conventional F-RAN architecture that exploits cloud resources for complex resource allocation tasks and local cache at the F-APs for fast data delivery to the UDs.

Complexity of I-RSRM: The computational complexity I-RSRM algorithm depends on the number of the required Pareto-optimal points. Using the reported computational complexity of Algorithms 3 and 4, the computational complexity of obtaining the two corner points of the Pareto-front is $\mathcal{O}\left(\Delta_{\max }^{(1)}\left(K L N+K^{2} N\right)\right)$. We assume that $\Delta_{\max }^{(2)}$ iterations are required for convergence of the Steps 5-9 of Algorithm 5. Hence, we can readily justify that to obtain a single Pareto-optimal point, I-RSRM requires $\mathcal{O}\left(\left(\Delta_{\max }^{(1)}+\Delta_{\max }^{(2)}\right)\left(K L N+K^{2} N\right)\right)$ iterations. Recall, I-RSRM obtains $N_{F}$ points of the Pareto-front. Therefore, the overall computational complexity of the proposed I-RSRM algorithm is $\mathcal{O}\left(N_{F}\left(\Delta_{\max }^{(1)}+\Delta_{\max }^{(2)}\right)\left(K L N+K^{2} N\right)\right)$.

Complexity of D-RSRM: The major computational complexity of D-RSRM algorithm is contributed by the Step 2 and the Steps 5-12 of Algorithm 6. For Step 2, Algorithm 6 requires $\mathcal{O}\left(K^{2} N+K L\right)$ operations. Let, $\Delta_{\max }^{(3)}$ iterations are required for convergence of the Steps 7-10 of 
Algorithm 6. Since only power allocations are used in the Steps 5-12 of Algorithm 6, the required number of operations for these steps is $\mathcal{O}\left(\Delta_{\max }^{(3)} K L N\right)$. Therefore, the overall computational complexity of the proposed D-RSRM algorithm to obtain $N_{F}$ points in the Pareto-front is obtained as $\mathcal{O}\left(N_{F} \Delta_{\max }^{(3)} K L N+K^{2} N\right)$. Evidently, D-RSRM brings substantial reduction of the computational complexity, especially for the system with large number of F-APs and RRBs.

\section{Simulation Results}

In this section, the performance of the proposed resource allocation algorithms is evaluated by simulations. For simulations, we consider a $300 m \times 300 m$ square F-RAN cell with one CBS in the center, 28 UDs, 10 F-APs, and 36 RRBs. The UDs are grouped into 7 device-clusters where cluster-1, cluster-2, cluster-3, cluster-4, cluster-5, cluster-6, and cluster-7 contain 5 UDs, 3 UDs, 3 UDs, 5 UDs, 4 UDs, 3 UDs, and 5 UDs, respectively. The F-APs are deployed on a circle of $35 \mathrm{~m}$ radius. The minimum distance from the F-APs to UDs and the minimum distance from the CBS to UDs are set as $40 \mathrm{~m}$ and $75 \mathrm{~m}$, respectively. An example of the considered simulation setting is provided in Fig. 2. In each device cluster, maximum $\left[\frac{M^{\prime}}{2}\right]$ UDs are considered as cache-miss UDs and remaining UDs are considered as cache-hit UDs where $M^{\prime}$ is the total number of UDs in the device-cluster In the ensuing simulation results, each simulation experiment is repeated 25 times with randomly chosen coordinates of the UDs at the device-clusters and locations of F-APs, and the average result is presented. The channel coefficients are generated by considering Rayleigh distributed fading and the 3GPP path loss model, given by, $131.1+42.8 \log _{10}(d) \mathrm{dB}$ (where $d$ is the distance in kilometers) [36]. Similar to [37], we consider $P_{\max }^{F A P}=P_{\max }^{C B S}=33$ $\mathrm{dBm}, P_{c}=0.2$ Watt, $\varepsilon=0.7, \sigma^{2}=-174 \mathrm{dBm}$. Finally, for selecting a suitable EE-SE pair by using Algorithm 7, we consider $\left\{w_{i}\right\}=1, \forall i \in\{1,2,3\}$, in (30a) and (30b). We consider the following benchmark schemes. For all the benchmark schemes, the operating EE-SE pair is selected by employing the method described in Section IV. C.

- Downlink multicasting: Downlink multicasting approach entirely eliminates the intradevice cluster interference, such as, interference among the cache-hit UDs and cache-miss UDs of each device-cluster. To apply multicasting in a given device-cluster, both the F-AP and CBS first combine the messages of the cache-hit UDs and cache-hit UDs, respectively. Then, the F-AP and CBS transmit the combined message over the RRBs using two rates as such the transmitted message from the F-AP and CBS are decodable to all the cache-hit and cache-miss UDs, respectively. To mitigate interference between F-AP and CBS in the device-clusters, transmission power of both F-AP and CBS are jointly optimized by using Algorithm 1. To mitigate inter device-cluster interference, Algorithm 2 is used to obtain the 


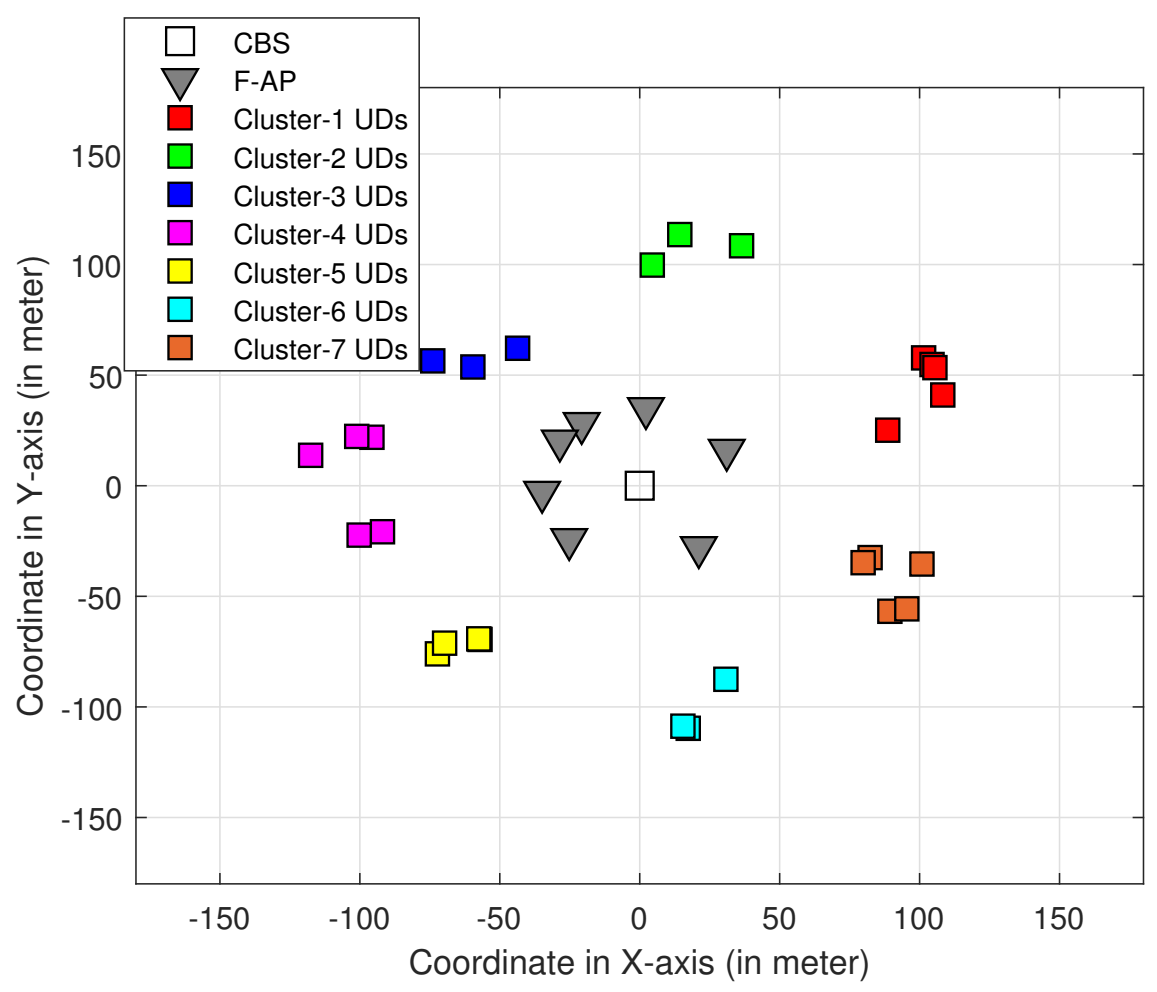

Fig. 2: An example of the considered simulation setting.

associations among device-clusters, F-APs, and RRBs.

- Unicast and treat interference as noise (Unicast/TIN): Unicast/TIN eliminates the interference between F-AP and CBS of the considered system model by allowing each device-cluster to be solely supported via CBS. However, this approach is subject to both inter device-cluster and intra device-cluster interference. To eliminate inter device-cluster interference, a many-to-one matching algorithm is applied to allocate orthogonal RRBs among the device-clusters. To mitigate intra device-cluster interference, an iterative function evaluation based power control is applied to allocate CBS's power over the RRBs and UDs.

- I-RSRM with iterative resource assignment (I-RSRM/IRA): I-RSRM/IRA applies similar resource allocation framework of the proposed I-RSRM algorithm. The only difference between I-RSRM and I-RSRM/IRA is that instead of 3D-resource matching, I-RSRM/IRA applies an alternating optimization technique, given in Algorithm 3 of [38], to determine the associations among device-clusters, F-APs, and RRBs.

\section{A. Effect of EE-SE Trade-off Control Parameter}

Figs. $3 \mathrm{a}$ and $3 \mathrm{~b}$ illustrate the EE and SE of the proposed I-RSRM algorithm for different values of the EE-SE trade-off control parameter $\alpha$. For all the considered device-clusters in 


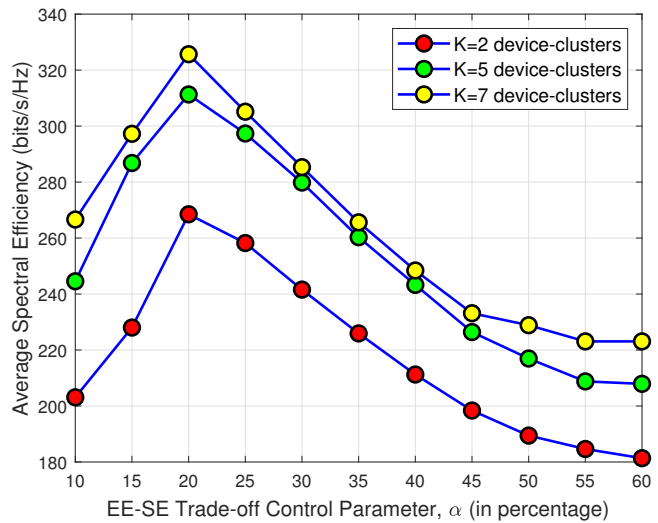

(a) SE versus (vs.) $\alpha$.

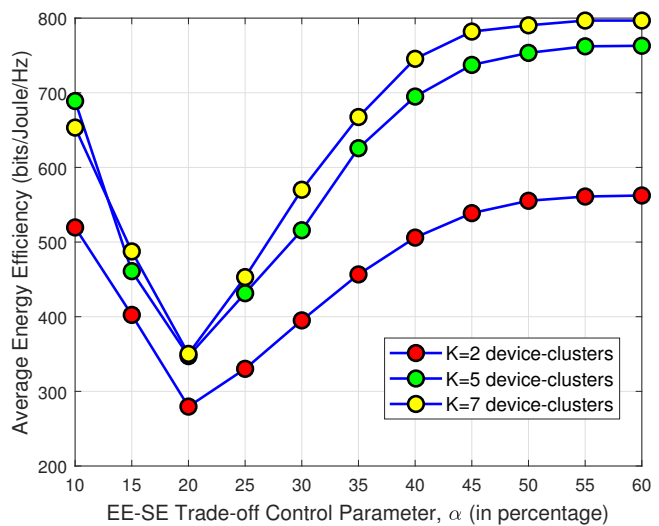

(b) EE versus (vs.) $\alpha$.

Fig. 3: Impact of EE-SE trade-off control parameter, $\alpha$, on the $\mathrm{EE}$ and $\mathrm{SE}$ of the proposed I-RSRM algorithm considering $L=3$ F-APs, $N=36$ RRBs, and $N_{R}=3$.

Figs. 3a and 3b, when $\alpha$ approaches small or large value, such as $\alpha=10 \%$ or $\alpha=60 \%$, a large system EE is achieved, and when $\alpha$ approaches moderate value, such as $\alpha=30 \%$, a large system SE is achieved. Moreover, for a given range of $\alpha$, the system EE and SE depict opposite behavior, i.e., increase of system SE results in decrease of system EE and vice versa. Such an observation is intuitive as EE and SE of the system can not be maximized simultaneously. An explanation of achieving large EE for both small and large values of $\alpha$ is provided as follows.

- Small $\alpha$ : When $\alpha \rightarrow 0$, the solutions to (28) and (29) approach the corner points of the developed Pareto-front. Particularly, the EE obtained by trading-off $\alpha$ percentage of optimal SE approaches the achievable EE of the system operating with maximum SE, and the SE obtained by trading-off $\alpha$ percentage of optimal EE approaches the achievable SE of the system operating with maximum EE. Essentially, as $\alpha \rightarrow 0, \boldsymbol{E} \boldsymbol{E}^{* *} \rightarrow \widehat{E E}_{\boldsymbol{S E}=\boldsymbol{S} \boldsymbol{E}^{*}}$ and $\boldsymbol{S} \boldsymbol{E}^{* *} \rightarrow \widehat{S E}_{\boldsymbol{E} \boldsymbol{E}=\boldsymbol{E} \boldsymbol{E}^{*}}$, and consequently, $\chi_{E E} \rightarrow 0$ and $\chi_{S E} \rightarrow 0$ are satisfied. We can illustrate that in the considered simulation setup, the relative EE gap between the two corner points of the developed Pareto-front is much larger than relative the SE gap. Hence, for small $\alpha, \frac{\boldsymbol{E} \boldsymbol{E}^{*}-\boldsymbol{E} \boldsymbol{E}^{* *}}{\boldsymbol{S} \boldsymbol{E}^{*}-\boldsymbol{S} \boldsymbol{E}^{* *}} \gg 1$ and $\mathcal{B O} \mathcal{T}_{2}>\mathcal{B O} \mathcal{T}_{1}$ are usually satisfied. Recall, when $\mathcal{B O T}_{2}>\mathcal{B O T}_{1}$ holds, the achievable EE and SE of the system are given by $\left(1-\frac{\alpha}{100}\right) \boldsymbol{E} \boldsymbol{E}^{*}$ and $\boldsymbol{S} \boldsymbol{E}^{* *}$, respectively. Since $\alpha$ is small, the system EE approaches the optimal EE, and owing to the conflicting behavior of EE and SE, SE becomes small. Accordingly, when $\alpha$ is small, the considered system example obtains large EE and small SE.

- Large $\alpha$ : When $\alpha$ is large, the SE and EE constraints of (28) and (29) are relaxed. As a result, $\boldsymbol{E} \boldsymbol{E}^{* *} \rightarrow \boldsymbol{E} \boldsymbol{E}^{*}$ and $\boldsymbol{S} \boldsymbol{E}^{* *} \rightarrow \boldsymbol{S} \boldsymbol{E}^{*}$ are satisfied. Therefore, eqs. (30a) and (30b) can be modified as $\mathcal{B O} \mathcal{T}_{1} \rightarrow \chi_{E E}-\alpha+\frac{(\alpha / 100)^{2}}{1-\alpha / 100}$ and $\mathcal{B O} \mathcal{T}_{2} \rightarrow \chi_{S E}-\alpha+\frac{(\alpha / 100)^{2}}{1-\alpha / 100}$, respectively. 


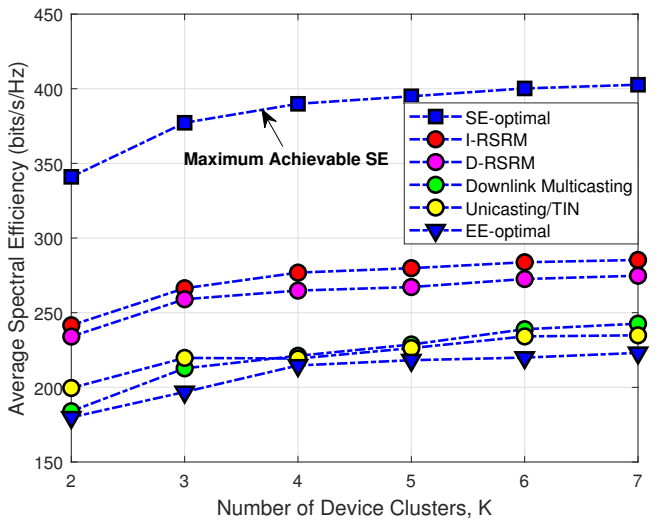

(a) SE vs. number of device-clusters for $\alpha=30 \%$.

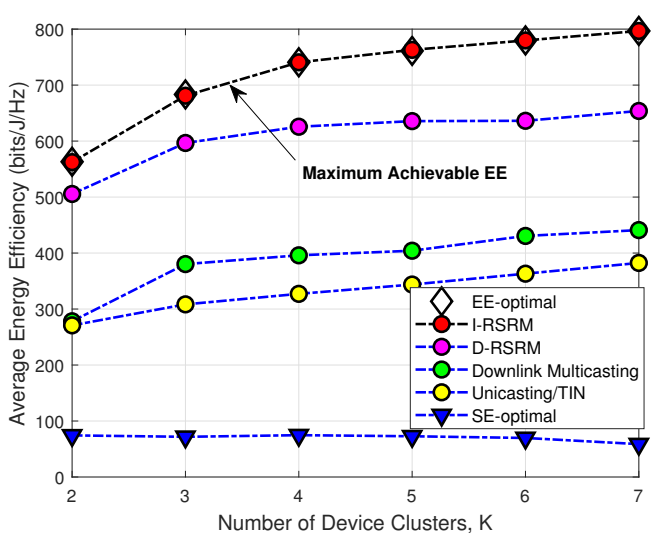

(b) EE vs. number of device-clusters for $\alpha=60 \%$.

Fig. 4: EE and SE comparison among the proposed and benchmark schemes for different number of device-clusters considering $L=3 \mathrm{~F}$-APs, $N=36 \mathrm{RRBs}$, and $N_{R}=3$.

Since the relative EE gap between the two corner points of the developed Pareto-front is much larger than the relative SE gap, $\chi_{E E}>\chi_{S E}$ and $\mathcal{B O} \mathcal{T}_{1}>\mathcal{B O} \mathcal{T}_{2}$ are satisfied. Recall, when $\mathcal{B O T}_{1}>\mathcal{B O} \mathcal{T}_{2}$ holds, the achievable EE of the system is given by $\boldsymbol{E} \boldsymbol{E}^{* *}$, and as mentioned earlier that for a large $\alpha, \boldsymbol{E} \boldsymbol{E}^{* *}$ approaches the optimal system EE. Consequently, the considered system example also achieves large EE as $\alpha$ becomes large.

Meanwhile, between the aforementioned two extreme cases, certain $\alpha$ does exist for which system chooses a strategy that obtains a near-optimal SE. Fig. 3a illustrates that as $\alpha$ takes a moderate value, i.e., $\alpha=30 \%$, for all the device-clusters, the considered system example achieves larger SE compared to the scenarios when $\alpha=10 \%$ and $\alpha=60 \%$. Note that $\alpha$ is a system defined control parameter. To determine a suitable value of $\alpha$, one can first conduct an offline simulation where system EE and SE are evaluated for different values of $\alpha$. Subsequently, by inspecting these results, the suitable value of $\alpha$ is selected so that the required design criteria are satisfied.

\section{B. EE and SE Comparison Among the Proposed and Baseline Schemes}

Figs. $4 \mathrm{a}$ and $4 \mathrm{~b}$ plot the achievable SE and EE of the proposed schemes for different deviceclusters while considering $\alpha=30 \%$ and $\alpha=60 \%$, respectively. SE and EE of the EE-optimal and SE-optimal schemes are also depicted in Figs. 4a and 4b, respectively. Performance of the EEoptimal and SE-optimal schemes is obtained from Algorithm 3 and Algorithm 4, respectively. The SE-optimal and EE-optimal schemes provide the two corner points of the Pareto-front developed by I-RSRM algorithm. Figs. $4 \mathrm{a}$ and $4 \mathrm{~b}$ depict that for all the device-clusters, the relative EE gap between the EE-optimal and SE-optimal schemes is much wider than the relative SE gap between EE-optimal and SE-optimal schemes. This confirms the previously mentioned fact, that is, the relative EE gap between the two corner points of the developed Pareto-front 
is much larger than the relative SE gap. Fig. 4a depicts that I-RSRM with $\alpha=30 \%$ achieves approximately $70 \%$ of the optimal SE which is intuitively expected. Particularly, by trading-off $30 \%$ of optimal SE, I-RSRM tremendously improves the achievable system EE. For instance, for $K=5$ device-clusters, I-RSRM with $\alpha=30 \%$ improves the system EE by 6.09 times compared to the SE-optimal scheme. Moreover, I-RSRM with $\alpha=30 \%$ achieves improved SE compared to the EE-optimal scheme as well. In Fig. 4 a, for $K=5$ device-clusters, I-RSRM with $\alpha=30 \%$ achieves $28.25 \%$ improved SE compared to the EE-optimal scheme. Finally, Fig. $4 \mathrm{~b}$ depicts that for all the device-clusters, I-RSRM with $\alpha=60 \%$ approaches EE-optimal scheme, and it is consistent with Fig. 3b. We observe that the conventional EE-optimal and SE-optimal schemes do not provide both SE and EE guarantee simultaneously. In contrast, by tuning $\alpha$, the proposed I-RSRM algorithm not only improves the trade-off between EE and SE but also can approach the solution provided by the EE-optimal and SE-optimal schemes. Hence, the proposed I-RSRM algorithm is more amenable compared to the conventional EE-optimal and SE-optimal schemes.

Figs. 4a and 4b also confirm the substantial SE and EE gain achieved by the proposed IRSRM algorithm. Note that both downlink multicasting and unicast/TIN approaches provide certain form of interference management for the considered system model, and consequently, they are the viable benchmark schemes for proposed resource allocation algorithms. Moreover, both of these approaches can be obtained as special cases of the proposed I-RSRM algorithm. Essentially, I-RSRM outperforms both of downlink multicasting and unicast/TIN approaches. Fig. 4a depicts that for $K=5$ device-clusters, I-RSRM achieves $22.36 \%$ and $23.65 \%$ improved SE compared to downlink multicasting and unicast/TIN approaches, respectively. Similarly, Fig. 4b depicts that for $K=5$ device-clusters, I-RSRM achieves $88.76 \%$ and $121.93 \%$ improved system EE compared to downlink multicasting and unicast/TIN approaches, respectively. In a downlink multicasting approach, to avoid intra device-cluster interference, only the minimum throughput of the cache-hit and cache-miss UDs in each device-cluster is targeted. The achievable throughput of such an approach is substantially reduced as the channel condition of the UD's with minimum throughput is deteriorated. Note that both downlink multicasting and I-RSRM use the same algorithm for 3D-resource matching. Essentially, compared to downlink multicasting, I-RSRM achieves performance gain by exploiting an optimized RS-CMD technique. On the other hand, compared to unicast/TIN, I-RSRM achieves performance gain by exploiting the simultaneous transmission from both F-APs and CBS and optimized power allocations to effectively suppress interference in the network. Overall, although the proposed I-RSRM algorithm allows more interference in the system than both downlink multicasting and unicast/TIN approaches, due 


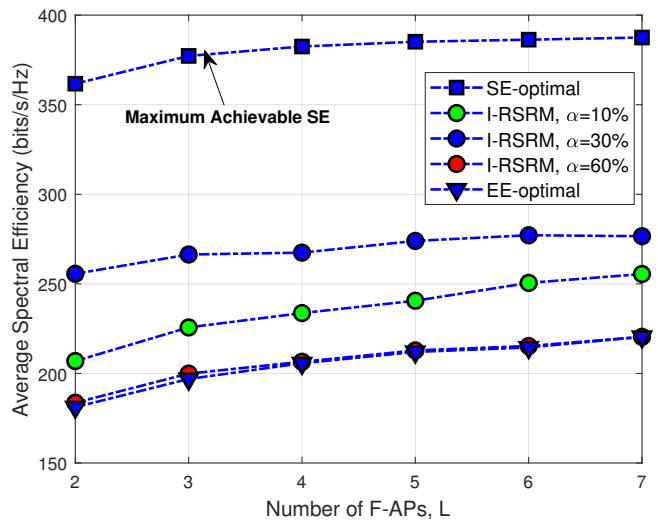

(a) SE vs. number of F-APs for $\alpha=10 \%, 30 \%, 60 \%$.

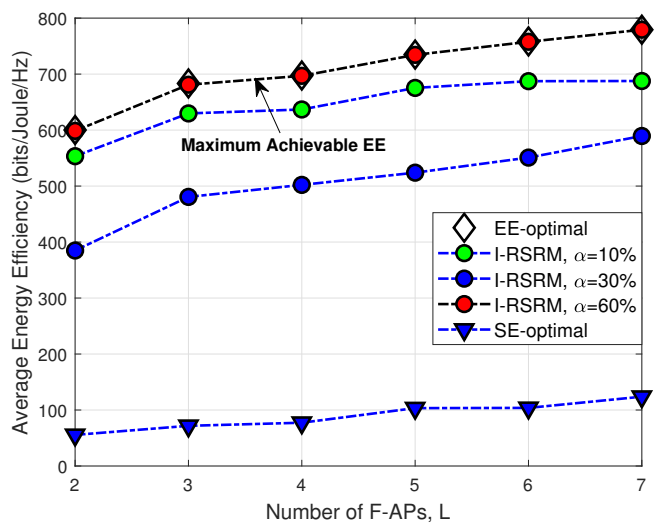

(b) EE vs. number of F-APs for $\alpha=10 \%, 30 \%, 60 \%$.

Fig. 5: EE and SE of the proposed I-RSRM algorithm for different number of F-APs considering $K=3$ device-clucters $N=36 \mathrm{RRRe}$ and $N_{R}=$ ?

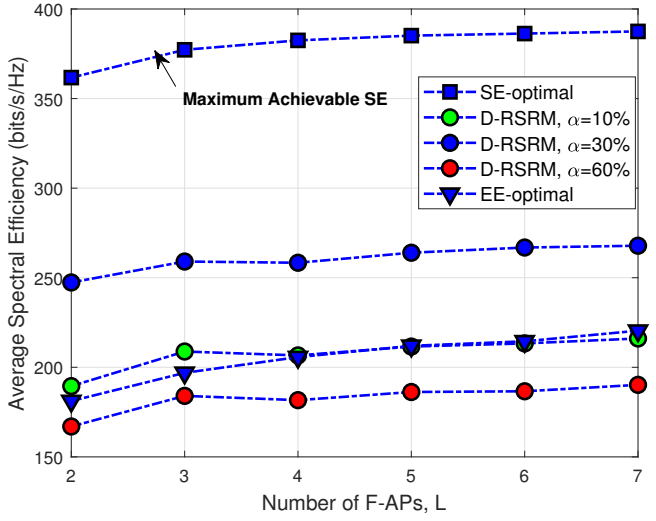

(a) SE vs. number of F-APs for $\alpha=10 \%, 30 \%, 60 \%$.

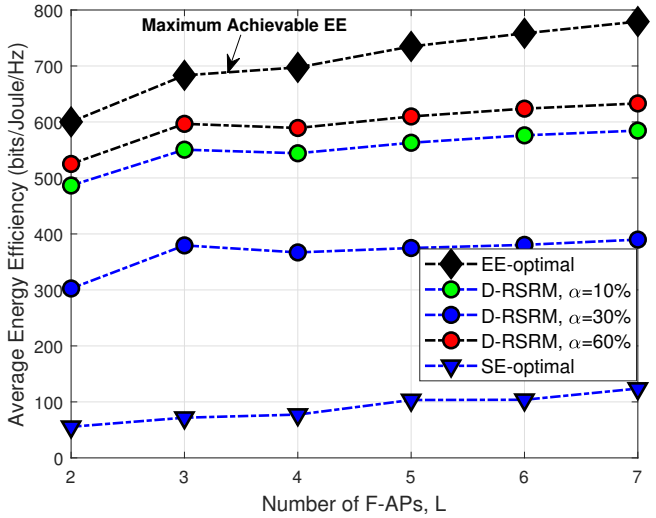

(b) EE vs. number of F-APs for $\alpha=10 \%, 30 \%, 60 \%$.

Fig. 6: EE and SE of the proposed D-RSRM algorithm for different number of F-APs considering $K=3$ device-clusters, $N=36 \mathrm{RRBs}$, and $N_{R}=3$.

to an optimized resource allocation, I-RSRM achieves considerable EE and SE gain over both downlink multicasting and unicast/TIN approaches. Fig. 4a also depicts that the proposed lowcomplexity D-RSRM algorithm achieves from $95 \%$ to $97 \%$ of SE of I-RSRM algorithm. However, Fig. 4b depicts that as the number of device-clusters in the network is increased, the EE gap between the proposed I-RSRM and D-RSRM algorithms is noticeably increased.

Figs. 5a and 5b illustrate variation of the SE and EE of the proposed I-RSRM algorithm for different number of F-APs, respectively. As the number of F-APs in the network is increased, the diversity gain obtained from the opportunistic assignment between device-clusters and F-APs is enhanced. Such a fact leads to the improvement of both SE and EE of the system. As expected, for all the F-APs, the SE (EE) of the proposed I-RSRM algorithm resides between SE (EE) of SE-optimal and EE-optimal schemes. Moreover, Figs. 5a and 5b depict that I-RSRM achieves maximum SE and EE for $\alpha=30 \%$ and $\alpha=60 \%$ which is consistent with the observation made 


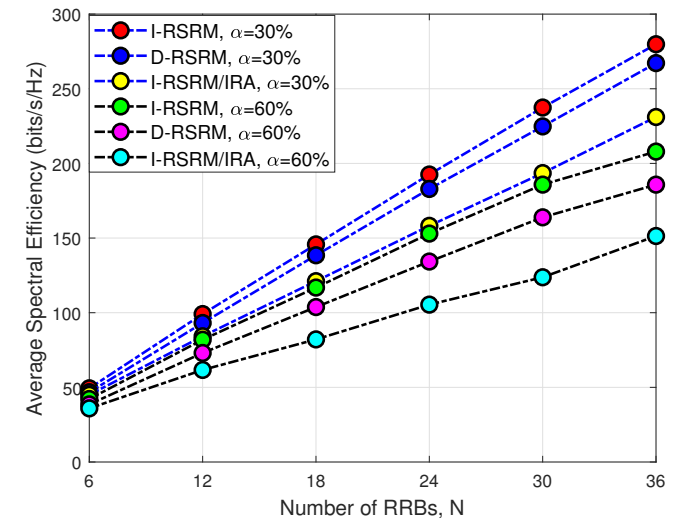

(a) SE vs. number of RRBs for $\alpha=30 \%$ and $\alpha=60 \%$.

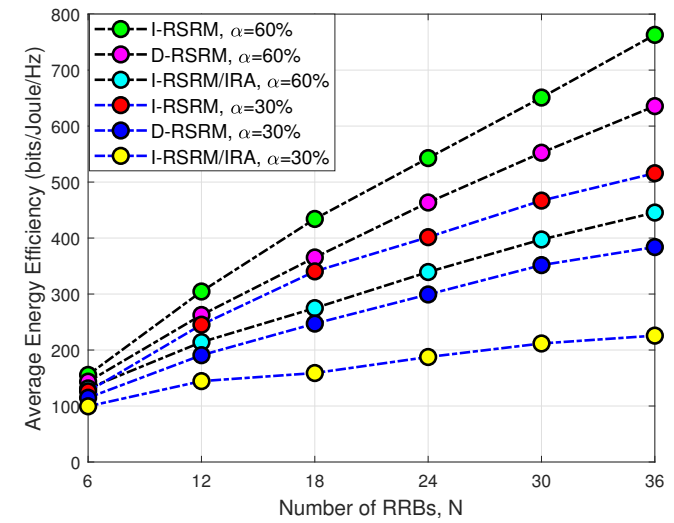

(b) EE vs. number of RRBs for $\alpha=30 \%$ and $\alpha=60 \%$.

Fig. 7: EE and SE comparison among I-RSRM, D-RSRM, and I-RSRM/IRA algorithms for different number of RRBs considering $K=5$ device-clusters, $L=3$ F-APs, and $N_{R}=3$.

from Figs. 3a and 3b, respectively. For $L=4$ F-APs, compared to SE-optimal scheme, I-RSRM with $\alpha=10 \%, 30 \%$, and $60 \%$ achieves $61.10 \%, 69.92 \%$, and $53.99 \%$ of optimal SE, respectively, and improves the achievable EE by 8.24, 6.49, and 9.01 times, respectively. Similarly, For $L=4$ F-APs, compared to EE-optimal scheme, I-RSRM with $\alpha=60 \%, 30 \%$, and $10 \%$ achieves $99.92 \%, 72 \%$, and $91.31 \%$ of optimal EE, respectively, and improves the achievable SE by 1.004 , 1.3, and 1.14 times, respectively. Indeed, the proposed I-RSRM algorithm improves the trade-off between EE and SE of both EE-optimal and SE-optimal schemes.

Figs. 6a and 6b plot SE and EE of the proposed D-RSRM algorithm for different number of F-APs, respectively. As expected, both SE and EE of D-RSRM algorithm are improved as the number of F-APs is increased. However, compared to I-RSRM, D-RSRM experiences higher SE and EE loss compared to SE-optimal and EE-optimal schemes, respectively. For example, for $L=4$ F-APs, I-RSRM can achieve up to $69.92 \%$ of optimal SE and $99.92 \%$ of optimal EE of the system. However, for the same system setting, D-RSRM can achieve up to $67.55 \%$ of optimal SE and $84.49 \%$ of optimal EE of the system. In addition, compared to I-RSRM, D-RSRM has inferior EE-SE trade-off performance. For example, for $L=4$ F-APs, I-RSRM can achieve up to $99.92 \%$ of EE of EE-optimal scheme while improving SE by 1.004 times. However, for the same system setting, D-RSRM can achieve up to $84.49 \%$ of EE of EE-optimal scheme while sacrificing $11.68 \%$ loss of SE. From the aforementioned discussion, it is evident that the gap between maximum achievable SE of I-RSRM and D-RSRM algorithms is small. Moreover, DRSRM has much lower computational complexity than I-RSRM. Hence, D-RSRM is a suitable alternative to I-RSRM for large size system given the system prioritizes SE. However, when the system prioritizes the improvement of the trade-off between EE and SE, I-RSRM is preferred.

Figs. 7a and 7b compare SE and EE of I-RSRM, D-RSRM, and I-RSRM/IRA algorithms for 


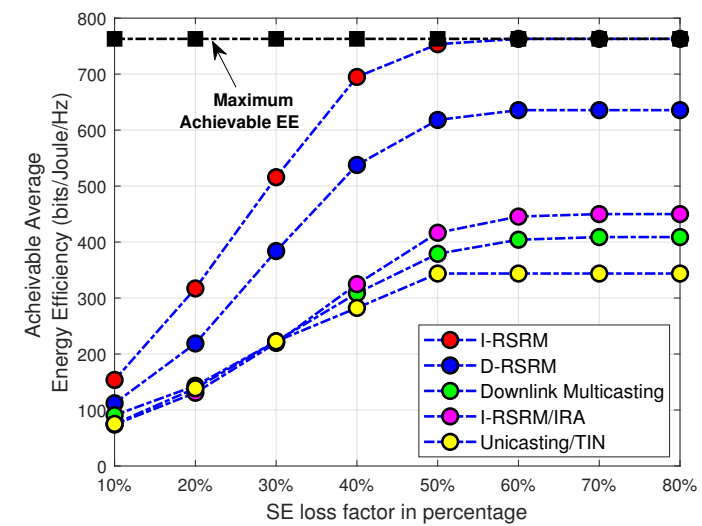

(a) SE loss-rate vs. achievable EE.

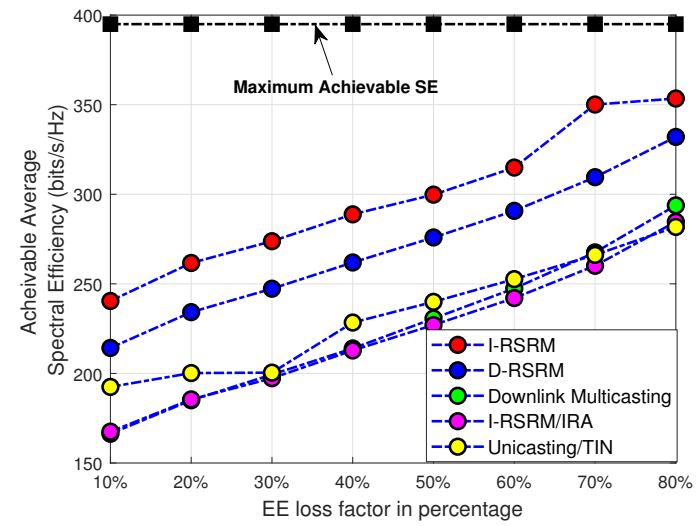

(b) EE loss-rate vs. achievable SE.

Fig. 8: EE vs. SE trade-off comparison among the proposed and benchmark schemes considering $K=5$ device-clusters, $L=3$ F-APs, $N_{R}=3$, and $N=36$ RRBs.

different RRBs. As expected, as the number of RRBs is increased, both SE and EE of all the considered schemes in Figs. 7a and 7b are increased. Note that unlike I-RSRM and D-RSRM, I-RSRM/IRA uses an alternating optimization technique given in [38, Algorithm 3] to obtain the assignments of RRBs and F-APs among the device-clusters. However, despite convergence, such an alternating optimization technique does not guarantee near-optimal solution to P2.6. Consequently, I-RSRM/IRA obtains an inferior matching among the device-clusters, F-APs, and RRBs, and such an inferior matching leads to the noticeable SE/EE loss compared to the proposed algorithms, especially for large RRBs. From Fig. 7a, at $N=30$ RRBs, the proposed I-RSRM and D-RSRM algorithms with $\alpha=30 \%$ improve the achievable SE of I-RSRM/IRA by $22.69 \%$ and $16.17 \%$, respectively. From Fig. 7 b, at $N=30$ RRBs, the proposed I-RSRM and D-RSRM algorithms with $\alpha=60 \%$ improve the achievable EE of I-RSRM/IRA by $63.78 \%$ and $38.98 \%$, respectively. Such an observation clearly demonstrates that the our proposed 3Dresource matching is highly efficient to improve both $\mathrm{SE}$ and $\mathrm{EE}$ of the system.

\section{EE (resp. SE) Loss-rate vs. SE (resp. EE) Improvement}

Figs. $8 \mathrm{a}$ and $8 \mathrm{~b}$ demonstrate EE and SE loss-rate vs. SE and EE improvement for the proposed and benchmark schemes, respectively. Each point on Figs. 8a and 8b provides the achievable EE and SE obtained by trading-off certain percentage of optimal SE and EE, respectively. We make the following three observations. First, as the SE loss-factor and EE loss-factor become asymptotically large, the achievable EE and SE of I-RSRM algorithm approach the EE and SE obtained by the EE-optimal and SE-optimal schemes, respectively. Second, for I-RSRM algorithm, the improvement of EE by trading-off optimal SE is much larger than the improvement of SE by trading-off optimal EE. For instance, when the SE loss-factor is increased from $20 \%$ to $50 \%$, the EE of I-RSRM algorithm is improved by $137.59 \%$ as depicted from Fig. 8 a. In contrast, 
when the EE loss-factor is increased from $20 \%$ to $50 \%$, the SE of I-RSRM algorithm is improved by $14.57 \%$ as depicted from Fig. 8 b. Finally, we observe that for a given SE and EE loss factor, the proposed I-RSRM and D-RSRM algorithms achieve substantially large EE and SE gain compared to the benchmark schemes. For instance, from Fig. 8a, when the system is willing to trade-off $30 \%$ of optimal SE, I-RSRM (resp. D-RSRM) achieves $131.45 \%, 131.67 \%$ and $134.61 \%$ (resp. $72.26 \%, 72.43 \%$, and $74.61 \%$ ) improved EE compared to the downlink multicasting, unicast/TIN, and I-RSRM/IRA schemes, respectively. Similarly, from Fig. 8b, when the system is willing to trade-off 30\% of optimal EE, I-RSRM (resp. D-RSRM) achieves $37.32 \%, 36.36 \%$ and $38.76 \%$ (resp. 24.03\%, 23.37\%, and 25.33\%) improved SE compared to the downlink multicasting, unicast/TIN, and I-RSRM/IRA schemes, respectively. The aforementioned observations suggest that compared to the benchmark schemes, both of the proposed I-RSRM and D-RSRM algorithms significantly improve the trade-off between EE and SE of the system.

\section{CONCLUSION}

We proposed cluster-based resource allocations to simultaneously maximize system EE and SE of the content distribution phase of RS-CMD integrated and EC enabled F-RAN. By adopting the $\epsilon$-constraint method, an EE-SE trade-off optimization was conducted while jointly performing the transmission power allocation of CBS and F-APs, assignment of the F-APs among the deviceclusters, and scheduling of the RRBs among the device-clusters. An IFE based power control method and 3D-resource matching were developed. Based on such analyses, near-optimal and sub-optimal algorithms were proposed to obtain the Pareto-front of EE-SE trade-off optimization problem, and select a suitable operating EE-SE pair. Simulation results confirmed the following two observations: (i) By tuning a suitable EE-SE trade-off control parameter, the proposed nearoptimal algorithm substantially improves the trade-off between EE and SE compared to the conventional EE-optimal and SE-optimal schemes, and (ii) the proposed near-optimal and suboptimal algorithms yield significant EE and SE gain compared to the benchmark schemes.

\section{APPENDIX A}

We assume that the tuple $z^{*}=\left(\boldsymbol{P}^{*}, \boldsymbol{Q}^{*}, \boldsymbol{a}^{*}, \boldsymbol{b}^{*}\right)$ is an optimal solution to P1. For a proof by contradiction, we consider that $z^{*}$ is not weakly Pareto-optimal to P0. Hence, another tuple $\tilde{\boldsymbol{z}} \triangleq(\hat{\boldsymbol{P}}, \hat{\boldsymbol{Q}}, \hat{\boldsymbol{a}}, \hat{\boldsymbol{b}})$ will exist for which $\operatorname{EE}(\tilde{\boldsymbol{z}})>\operatorname{EE}\left(\boldsymbol{z}^{*}\right)$ and $\operatorname{SE}(\tilde{\boldsymbol{z}})>\operatorname{SE}\left(\boldsymbol{z}^{*}\right)$ will be satisfied. Moreover, since $z^{*}$ is a solution to $\mathrm{P} 1$, we obtain $\operatorname{SE}(\tilde{z})>\operatorname{SE}\left(z^{*}\right) \geq \epsilon$. Hence, $\tilde{z}$ is also a feasible solution to P1, and it improves objective function of P1 compared to $z^{*}$. Essentially, $z^{*}$ can not be an optimal solution to $\mathrm{P} 1$ which is contradictory to our initial assumption. Hence, an optimal solution to P1 is weakly Pareto-optimal to P0. 


\section{APPENDIX B}

According to the Karush-Khun-Tucker (KKT) optimality conditions, the first order derivative of $\mathcal{L}_{j}$ with respect to the power allocation variables will be zero. By setting $\frac{\partial \mathcal{L}_{j}}{\partial P_{l, n}^{C, j}}=0$, we obtain

$$
\frac{1}{1+\gamma_{l, n}^{\boldsymbol{C}, j}} \frac{\partial \gamma_{l, n}^{\boldsymbol{C}, j}}{\partial P_{l, n}^{C, j}}+\frac{1}{1+\widetilde{\gamma}_{n}^{\boldsymbol{C}, j}} \frac{\partial \widetilde{\gamma}_{n}^{\boldsymbol{C}, j}}{\partial P_{l, n}^{C, j}}+\sum_{e \in \mathcal{S}_{j}^{(L)}} \frac{1}{1+\gamma_{l, e, n}^{\boldsymbol{p}, j}} \frac{\partial \gamma_{l, e, n}^{\boldsymbol{p}, j}}{\partial P_{l, n}^{C, j}}+\sum_{t \in \mathcal{S}_{j}^{(H)}} \frac{1}{1+\widetilde{\gamma}_{t, n}^{\boldsymbol{p}, j}} \frac{\partial \widetilde{\gamma}_{t, n}^{\boldsymbol{p}, j}}{\partial P_{l, n}^{C, j}}=\frac{\eta+\lambda_{l}}{\varepsilon / \ln 2}
$$

By plugging (2)-(5) to (32) and performing some algebraic manipulations, we obtain

$$
P_{l, n}^{C, j}=\frac{\gamma_{l, n}^{C, j} /\left(1+\gamma_{l, n}^{C, j}\right)}{\frac{\eta / \varepsilon+\lambda_{l} / \varepsilon}{1 / \ln 2}+\frac{\left(\widetilde{\gamma}_{n}^{C, j}\right)^{2}\left|h_{\hat{k}_{j}, n}^{(l)}\right|^{2}}{\left(1+\widetilde{\gamma}_{n}^{C, j}\right) Q_{n}^{C, j}\left|h_{\tilde{k}_{j}, n}^{(0)}\right|^{2}}+\sum_{t \in \mathcal{S}_{j}^{(H)}} \frac{\left(\widetilde{\gamma}_{t, n}^{p, j}\right)^{2}\left|h_{t, n}^{(l)}\right|^{2}}{\left(1+\widetilde{\gamma}_{t, n}^{\boldsymbol{p}, j}\right) Q_{t, n}^{p, j}\left|h_{t, n}^{(0)}\right|^{2}}} .
$$

Eq. (33) provides the optimality condition for $P_{l, n}^{C, j}$. Similarly, the optimality conditions for the other power allocation variables, namely, $Q_{n}^{C, j}, P_{l, e, n}^{p, j}$, and $Q_{l, n}^{p, j}$, are respectively obtained as

$$
\begin{aligned}
& Q_{n}^{C, j}=\frac{\widetilde{\gamma}_{n}^{C, j} /\left(1+\widetilde{\gamma}_{n}^{C, j}\right)}{\frac{\eta / \varepsilon+\nu / \varepsilon}{1 / \ln 2}+\frac{\left(\gamma_{l, n}^{C, j}\right)^{2}\left|h_{k_{j}, n}^{(0)}\right|^{2}}{\left(1+\gamma_{l, n}^{C, j}\right) P_{l, n}^{C, j}\left|h_{k_{j}, n}^{(l)}\right|^{2}}+\sum_{e \in \mathcal{S}_{j}^{(L)}} \frac{\left(\gamma_{l, e, n}^{\boldsymbol{p}, j}\right)^{2}\left|h_{e, n}^{(0)}\right|^{2}}{\left(1+\gamma_{l, e, n}^{\boldsymbol{p}, j}\right) P_{l, e, n}^{p, j}\left|h_{e, n}^{(l)}\right|^{2}}} \\
& P_{l, e, m}^{p, j}=\frac{\gamma_{l, e, n}^{p, j} /\left(1+\gamma_{l, e, n}^{p, j}\right)}{\frac{\eta+\lambda_{l}}{\varepsilon / \ln 2}+\frac{\left(\gamma_{l, n}^{C, j}\right)^{2}}{\left(1+\gamma_{l, n}^{C, j}\right) P_{l, n}^{C, n}}+\sum_{\begin{array}{c}
e^{\prime} \in \mathcal{S}_{j}^{(L)} \\
e^{\prime} \neq e
\end{array}} \frac{\left(\gamma_{l, e^{\prime}, n}^{p}\right)^{2}}{\left(1+\gamma_{l, e^{\prime}, n}^{p, j}\right) P_{l, e^{\prime}, n}^{p, j}}+\frac{\left(\widetilde{\gamma}_{n}^{C, j}\right)^{2}\left|h_{\tilde{k}_{j}, n}^{(l)}\right|^{2}}{\left(1+\widetilde{\gamma}_{n}^{C, j}\right) Q_{n}^{C, j}\left|h_{\tilde{k}_{j}, n}^{(0)}\right|^{2}}+\sum_{t \in \mathcal{S}_{j}^{(H)}} \frac{\left(\widetilde{\gamma}_{t, n}^{p, j}\right)^{2}\left|h_{t, n}^{(l)}\right|^{2}}{\left(1+\widetilde{\gamma}_{t, n}^{\boldsymbol{p}, j}\right) Q_{t, n}^{p, j}\left|h_{t, n}^{(0)}\right|^{2}}} \\
& Q_{t, n}^{p, j}=\frac{\widetilde{\gamma}_{t, n}^{p, j} /\left(1+\widetilde{\gamma}_{t, n}^{p, j}\right)}{\frac{\eta+\nu}{\varepsilon / \ln 2}+\frac{\left(\widetilde{\gamma}_{n}^{C, j}\right)^{2}}{\left(1+\widetilde{\gamma}_{n}^{C, j}\right) Q_{n}^{C, j}}+\sum_{\substack{t^{\prime} \in \mathcal{S}_{j}^{(H)} \\
t^{\prime} \neq t}} \frac{\left(\widetilde{\gamma}_{t^{\prime}, n}^{p, j}\right)^{2}}{\left(1+\widetilde{\gamma}_{t^{\prime}, n}^{\boldsymbol{p}, j}\right) Q_{t^{\prime}, n}^{p, j}}+\frac{\left(\gamma_{l, n}^{C, j}\right)^{2}\left|h_{k_{j}, n}^{(0)}\right|^{2}}{\left(1+\gamma_{l, n}^{C, j}\right) P_{l, n}^{C, j}\left|h_{k_{j}, n}^{(l)}\right|^{2}}+\sum_{e \in \mathcal{S}_{j}^{(L)}} \frac{\left(\gamma_{l, e, n}^{p, j}\right)^{2}\left|h_{e, n}^{(0)}\right|^{2}}{\left(1+\gamma_{l, e, n}^{\boldsymbol{p}, j}\right) P_{l, e, n}^{p, j}\left|h_{e, n}^{(l)}\right|^{2}}} .
\end{aligned}
$$

Since the closed-from power allocation expressions are intractable, we use (33)-(36) to iteratively update the power allocation variables. To this end, we denote $\left\{\widehat{P}_{l, n}^{C, j}, \widehat{P}_{l, e, n}^{p, j}, \widehat{Q}_{n}^{C, j}, \widehat{Q}_{t, n}^{p, j}\right\}$ as the given power allocations. Thereupon, by replacing $\left\{P_{l, n}^{C, j}, P_{l, e, n}^{p, j}, Q_{n}^{C, j}, Q_{t, n}^{p, j}\right\}$ of (33)-(36) with $\left\{\widehat{P}_{l, n}^{C, j}, \widehat{P}_{l, e, n}^{p, j}, \widehat{Q}_{n}^{C, j}, \widehat{Q}_{t, n}^{p, j}\right\}$, we obtain the power allocation update equations of Proposition 1.

\section{APPENDix C}

The convergence of an iterative function evaluation (IFE) based power allocation for asymptotically high signal-to-inference-plus-noise-ratio (SINR) regime was justified in [39]. We present 
an alternative proof by using game theory and without resorting to the asymptotically high SNR assumption. To this end, we describe power allocation in the $j$-th device-cluster, $\forall j$, as a non-cooperative power control game (NCPCG) where the transmitted common messages and private messages are the selfish and rational (virtual) players. The NCPCG is defined by the tuple $\mathcal{G}=\left(\{I\},\left\{\Gamma_{I}\right\},\left\{\Pi_{I}\right\}\right)$, and its description is given as follows.

- $\{I\}$ is the set of the players in the game and the considered game has total $\left|\mathcal{S}_{j}^{(H)}\right|+$ $\left|\mathcal{S}_{j}^{(L)}\right|+2$ players. In particular, $I=1$ denotes the transmitted common message for the cache-hit UDs; $I=2$ denotes the transmitted common message for the cache-miss UDs; $I=3, \cdots\left|\mathcal{S}_{j}^{(L)}\right|+2$ denote the transmitted private messages for the cache-hit UDs; and $I=\left|\mathcal{S}_{j}^{(L)}\right|+3, \cdots\left|\mathcal{S}_{j}^{(L)}\right|+\left|\mathcal{S}_{j}^{(H)}\right|+2$ denote the transmitted private messages for the cache-miss UDs.

- $\Gamma_{I}$ is the strategy space set for the $I$-th player. Particularly, $\Gamma_{1}=\left\{P_{l, n}^{C, j} \mid P_{l, n}^{C, j} \geq 0\right\} ; \Gamma_{2}=$ $\left\{Q_{n}^{C, j} \mid Q_{n}^{C, j} \geq 0\right\}$; for $I=3, \cdots,\left|\mathcal{S}_{j}^{(L)}\right|+2, \Gamma_{I}=\left\{P_{l, e, n}^{C, j} \mid P_{l, e, n}^{C, j} \geq 0\right\}$ where $e=I-2$; and for $I=\left|\mathcal{S}_{j}^{(L)}\right|+3, \cdots,\left|\mathcal{S}_{j}^{(L)}\right|+\left|\mathcal{S}_{j}^{(H)}\right|+2, \Gamma_{I}=\left\{Q_{t, n}^{C, j} \mid Q_{t, n}^{C, j} \geq 0\right\}$ where $t=I-\left|\mathcal{S}_{j}^{(L)}\right|-2$.

- $\Pi_{I}$ is the utility function for the $I$-th player and for different $I$, utility functions are defined as (A.1)-(A.4) at the top of next page. In (A.1)-(A.4), $\boldsymbol{P}_{-I}$ denotes the power allocation strategy of all the players in the game except the $I$-th player.

Note that the utility function of the $I$-th player, $\forall I$, is continuous and differentiable in the strategy space. For each utility function, the first term provides the reward for improving the achievable data rate, the last term provides the cost of spending transmission power to improve data rate, and the remaining terms provide the reward (penalty) for creating less (high) interference in the network. We can depict that the utility function of the $I$-th player, $\forall I$, behaves similar to a quasi-concave function. Essentially, the considered NCPCG must exhibit a Nashequilibrium (NE) power allocation strategy [40, Theorem 3.2]. Under an NE power allocation strategy, no player can further improve its utility by choosing an alternative strategy, and thus, power allocation converges to a stable point. Note that for the quasi-concave utility functions, $\mathrm{NE}$ is achieved at the maximal points of the players' utility functions. It can be readily verified that power allocation update equations in Proposition 1 of our revised manuscript are obtained at the maximal points of the utility functions in (A.1)-(A.4). Hence, the power allocations given in Proposition 1 guarantee the achievement of NE for the players in NCPCG $\mathcal{G}$. In other words, the power allocations obtained from Algorithm 1 must converge to a stable solution to P2.4 regardless of the asymptotically high SINR assumption. We also emphasize that utility function of each player in the considered NCPCG incorporates social welfare through considering impact 


$$
\begin{aligned}
& \Pi_{1}\left(\Gamma_{1} \mid \boldsymbol{P}_{-1}\right)=\sum_{l=1}^{L} \sum_{n=1}^{N} a_{l, j} b_{l, j, n}\left(R_{l, n}^{\boldsymbol{C}, j}+\widetilde{R}_{n}^{\boldsymbol{C}, j}+\sum_{t \in \mathcal{S}_{j}^{(H)}} \widetilde{R}_{t, n}^{\boldsymbol{p}, j}-\frac{\eta+\lambda_{l}}{\varepsilon} P_{l, n}^{C, j}\right)^{\text {SUBMr }} \\
& \Pi_{2}\left(\Gamma_{2} \mid \boldsymbol{P}_{-2}\right)=\sum_{l=1}^{L} \sum_{n=1}^{N} a_{l, j} b_{l, j, n}\left(\widetilde{R}_{n}^{C, j}+R_{l, n}^{C, j}+\sum_{e \in \mathcal{S}_{j}^{(L)}} R_{l, e, n}^{b m P, j}-\frac{\eta+\nu}{\varepsilon} Q_{n}^{C, j}\right) \\
& \Pi_{I}\left(\Gamma_{I} \mid \boldsymbol{P}_{-I}\right)=\sum_{l=1}^{L} \sum_{n=1}^{N} a_{l, j} b_{l, j, n}\left(R_{l, I-2, n}^{\boldsymbol{p}, j}+\sum_{\substack{e^{\prime} \in \mathcal{S}_{j}^{(L)} \\
e^{\prime} \neq I-2}} R_{l, e^{\prime}, n}^{\boldsymbol{P}, j}+R_{l, n}^{\boldsymbol{C , j}}+\widetilde{R}_{n}^{\boldsymbol{C}, j}\right. \\
& \left.+\sum_{t \in \mathcal{S}_{j}^{(H)}} R_{t, n}^{p, j}-\frac{\eta+\lambda_{l}}{\varepsilon} P_{l, I-2, n}^{p, j}\right), I=3, \cdots,\left|\mathcal{S}_{j}^{(L)}\right|+2 \\
& \Pi_{I}\left(\Gamma_{I} \mid \boldsymbol{P}_{-I}\right)=\sum_{l=1}^{L} \sum_{n=1}^{N} a_{l, j} b_{l, j, n}\left(\widetilde{R}_{\begin{array}{c}
\text { p,j } \\
I-\left|\mathcal{S}_{j}^{(L)}\right|-2, n
\end{array}}+\sum_{\substack{t^{\prime} \in \mathcal{S}_{j}^{(H)} \\
t^{\prime} \neq I-\left|\mathcal{S}_{j}^{(L)}\right|-2}} \widetilde{R}_{t^{\prime}, n}^{P, j}+R_{l, n}^{\boldsymbol{C}, j}+\widetilde{R}_{n}^{\boldsymbol{C}, j}\right. \\
& \left.+\sum_{e \in \mathcal{S}_{j}^{(L)}} R_{l, e, n}^{\boldsymbol{p}, j}-\frac{\eta+\nu}{\varepsilon} Q_{I-\left|\mathcal{S}_{j}^{(L)}\right|-2, n}^{p, j}\right), I=\left|\mathcal{S}_{j}^{(L)}\right|+3, \cdots,\left|\mathcal{S}_{j}^{(L)}\right|+\left|\mathcal{S}_{j}^{(H)}\right|+2 .
\end{aligned}
$$

of creating interference in the network, and as a result, the NE of such a game indeed ensures a social optimal outcome [41]. Accordingly, Algorithm 1 converges to a stable and near-optimal solution to P2.4.

\section{APPENDIX D}

Assume that $\Phi^{*}$ is not Pareto-efficient. Consequently, $\Phi^{*}$ will contain at least two Paretoimprovement device-cluster pairs. Let $\mathcal{S}_{j}$ and $\mathcal{S}_{j}^{\prime}$ be the Pareto-improvement device-cluster pairs where $\boldsymbol{\Phi}^{*}(l, n)=j$ and $\boldsymbol{\Phi}^{*}\left(l^{\prime}, n^{\prime}\right)=j^{\prime}$. Since these two device-cluster pairs are Paretoimprovement pair, each of them will prefer the resources matched with the other one compared to their current matching. Hence, we can have at least one of the following three scenarios: (S1) $\left(l^{\prime}, n^{\prime}\right) \succ_{j}(l, n)$ and $(l, n) \succ_{j^{\prime}}\left(l^{\prime}, n^{\prime}\right),(\mathbf{S 2})\left(l^{\prime}, n\right) \succ_{j}(l, n)$ and $\left(l, n^{\prime}\right) \succ_{j^{\prime}}\left(l^{\prime}, n^{\prime}\right)$, and (S3) $\left(l, n^{\prime}\right) \succ_{j}(l, n)$ and $\left(l^{\prime}, n\right) \succ_{j^{\prime}}\left(l^{\prime}, n^{\prime}\right)$. For proving Proposition 3, it is suffice to show that $\boldsymbol{\Phi}^{*}$ does not contain two device-clusters for which the conditions S1-S3 hold true. Specifically, we 
need to show that the conditions S1-S3 do not hold true for the matching(s) obtained in any phase of Algorithm 2. Note that, in $\Phi^{*}$, the $j$-th and the $j^{\prime}$-th device-clusters are matched with the $l$-th and the $l^{\prime}$-th F-APs at the FM phase of Algorithm 2, respectively. However, the $n$-th and the $n^{\prime}$-th RRBs can be matched with the $j$-th and the $j^{\prime}$-th device-clusters, respectively, in either the FM phase, the RM phase, or the RS phase of Algorithm 2.

Let the $n$-th and the $n^{\prime}$-th RRBs be matched with the $j$-th and the $j^{\prime}$-th device-clusters, respectively, in the FM phase of Algorithm 2. We assume that the $j$-th and $j^{\prime}$-th device-clusters select their preferred pairs in two consecutive iterations of the FM phase of Algorithm 2, and the $j$-the device-cluster has a larger eligibility score than the $j^{\prime}$-th device-cluster. We need to consider the following three possible cases: (i) Both the $j$-th and $j^{\prime}$-th device-clusters prefer to be matched with the $(l, n)$ pair; (ii) the $j$-th and $j^{\prime}$-th device-clusters prefer to be matched with the $(l, n)$ and $\left(l, n^{\prime}\right)$ pairs, respectively; and (ii) the $j$-th and $j^{\prime}$-th device-clusters prefer to be matched with the $(l, n)$ and $\left(l^{\prime}, n\right)$ pairs, respectively. Capitalizing the Steps 5 and 6 of Algorithm 2, for these three cases, we obtain, respectively,

$$
\begin{aligned}
& \widehat{U}_{l, n}^{(j)}-\widehat{U}_{l^{\prime}, n^{\prime}}^{(j)}>\widehat{U}_{l, n}^{\left(j^{\prime}\right)}-\widehat{U}_{l^{\prime}, n^{\prime}}^{\left(j^{\prime}\right)} \Longrightarrow \widehat{U}_{l, n}^{(j)}+\widehat{U}_{l^{\prime}, n^{\prime}}^{\left(j^{\prime}\right)}>\widehat{U}_{l^{\prime}, n^{\prime}}^{(j)}+\widehat{U}_{l, n}^{\left(j^{\prime}\right)} \\
& \widehat{U}_{l, n}^{(j)}-\widehat{U}_{l^{\prime}, n}^{(j)}>\widehat{U}_{l, n^{\prime}}^{\left(j^{\prime}\right)}-\widehat{U}_{l^{\prime}, n^{\prime}}^{\left(j^{\prime}\right)} \Longrightarrow \widehat{U}_{l, n}^{(j)}+\widehat{U}_{l^{\prime}, n^{\prime}}^{\left(j^{\prime}\right)}>\widehat{U}_{l^{\prime}, n}^{(j)}+\widehat{U}_{l, n^{\prime}}^{\left(j^{\prime}\right)} \\
& \widehat{U}_{l, n}^{(j)}-\widehat{U}_{l, n^{\prime}}^{(j)}>\widehat{U}_{l^{\prime}, n}^{\left(j^{\prime}\right)}-\widehat{U}_{l^{\prime}, n^{\prime}}^{\left(j^{\prime}\right)} \Longrightarrow \widehat{U}_{l, n}^{(j)}+\widehat{U}_{l^{\prime}, n^{\prime}}^{\left(j^{\prime}\right)}>\widehat{U}_{l, n^{\prime}}^{(j)}+\widehat{U}_{l^{\prime}, n}^{\left(j^{\prime}\right)} .
\end{aligned}
$$

However, if $\mathbf{S 1}$ holds true, $\widehat{U}_{l^{\prime}, n^{\prime}}^{(j)}+\widehat{U}_{l, n}^{\left(j^{\prime}\right)}>\widehat{U}_{l, n}^{(j)}+\widehat{U}_{l^{\prime}, n^{\prime}}^{\left(j^{\prime}\right)}$ has to be satisfied; if $\mathbf{S} 2$ holds true, $\widehat{U}_{l^{\prime}, n}^{(j)}+\widehat{U}_{l, n^{\prime}}^{\left(j^{\prime}\right)}>\widehat{U}_{l, n}^{(j)}+\widehat{U}_{l^{\prime}, n^{\prime}}^{\left(j^{\prime}\right)}$ has to be satisfied; and if S3 holds true, $\widehat{U}_{l, n^{\prime}}^{(j)}+\widehat{U}_{l^{\prime}, n}^{\left(j^{\prime}\right)}>\widehat{U}_{l, n}^{(j)}+\widehat{U}_{l^{\prime}, n^{\prime}}^{\left(j^{\prime}\right)}$ has to be satisfied. Essentially, S1, S2, and $\mathbf{S 3}$ are in contradiction with (A.5), (A.6), and (A.7), respectively. Thus, when the $j$-th and $j^{\prime}$-th device-clusters are chosen to select their preferred pairs in two consecutive iterations of the FM phase of Algorithm 2, exchanging their matched resource(s) with each other is not beneficial. We can readily extend such a conclusion for any two device-clusters that are not necessarily chosen to select their preferred pairs in two consecutive iterations of the FM phase of Algorithm 2. Therefore, the conditions S1-S3 do not hold true for the matching(s) obtained in the FM phase of Algorithm 2.

Next, we assume that the $n$-th and the $n^{\prime}$-th RRBs are matched with the $j$-th and the $j^{\prime}$-th device-clusters, respectively, either at the RM or RS phase of Algorithm 2. We only need to show that the condition $\mathbf{S 3}$ does not hold true in both scenarios. In particular, if the considered RRBs are matched with the device-clusters at the RM phase of Algorithm 2, as per the Step 10 of Algorithm 2, we obtain $j=\arg \max _{\tilde{j} \in \mathcal{S}} \widehat{U}_{l, n}^{(\tilde{j})}$ and $j^{\prime}=\arg \max _{\tilde{j} \in \mathcal{S}} \widehat{U}_{l^{\prime}, n^{\prime}}^{(\tilde{j})}$. Obviously, $\widehat{U}_{l, n}^{(j)}>\widehat{U}_{l^{\prime}, n}^{\left(j^{\prime}\right)}$ and $\widehat{U}_{l^{\prime}, n^{\prime}}^{\left(j^{\prime}\right)}>\widehat{U}_{l, n^{\prime}}^{(j)}$ hold true, and consequently, $\widehat{U}_{l, n}^{(j)}+\widehat{U}_{l^{\prime}, n^{\prime}}^{\left(j^{\prime}\right)}>\widehat{U}_{l, n^{\prime}}^{(j)}+\widehat{U}_{l^{\prime}, n}^{\left(j^{\prime}\right)}$ hold true. On the other 
hand, if the $\left(n, n^{\prime}\right)$-th RRBs are swapped between the $j$-th and $j^{\prime}$-th device clusters at the RS phase of Algorithm 2, as per the Steps (14) and (15) of Algorithm 2, $\widehat{U}_{l, n}^{(j)}+\widehat{U}_{l^{\prime}, n^{\prime}}^{\left(j^{\prime}\right)}>\widehat{U}_{l, n^{\prime}}^{(j)}+\widehat{U}_{l^{\prime}, n}^{\left(j^{\prime}\right)}$ must be satisfied. Therefore, the condition $\mathbf{S 3}$ does not hold true for the matching(s) obtained in both RM and RS phases of Algorithm 2.

The aforementioned analysis depicts that both the $j$-th and $j^{\prime}$-th device-clusters can not strictly improve their overall utility by partially or completely exchanging their matched resources. Therefore, no two device-clusters in $\Phi^{*}$ has an incentive to exchange their matched resources with each other. Accordingly, $\Phi^{*}$ does not contain Pareto-improvement device-cluster pair, and as a result, $\Phi^{*}$ provides Pareto-efficient matching for the device-clusters.

\section{APPENDIX E}

Suppose, the final matching obtained by Algorithm 2, $\Phi^{*}$, does not provide a near-optimal solution to P2.6. Thus, another matching, $\Phi^{* *}$, exists that provides an improved overall utility for the device-clusters compared to $\Phi^{*}$. However, it is already proved that $\Phi^{*}$ is Pareto-efficient for the device-clusters. Essentially, it is not possible to strictly improve the overall utility of the device-clusters by exchanging the already matched resources among themselves. In other words, the assignment solution obtained from $\Phi^{* *}$ can not strictly improve the objective function of P2.6 compared to the assignment solution obtained from $\Phi^{*}$. Hence, for the given power allocations, $\Phi^{*}$ must converge to a near-optimal solution to P2.6.

\section{APPENDIX F}

Near-optimality of Algorithm 3: We first prove that the system EE is monotonically increased at each iteration of Algorithm 3. To this end, let $\left(\boldsymbol{P}^{(d)}, \boldsymbol{Q}^{(d)}, \boldsymbol{a}^{(d)}, \boldsymbol{b}^{(d)}\right)$ and $\left(\boldsymbol{P}^{(d+1)}, \boldsymbol{Q}^{(d+1)}, \boldsymbol{a}^{(d+1)}, \boldsymbol{b}^{(d+1)}\right)$ be the block of resource allocation variables at the $d$-th and $(d+1)$-th iteration of Algorithm 3. Let, $\eta^{(d)}=\frac{\sum_{j=1}^{K} \sum_{l=1}^{L} \sum_{n=1}^{N} a_{l, j}^{(d)} b_{n, l, j}^{(d)} \mathrm{T}_{j}^{(l, n)}\left(\boldsymbol{P}^{(d)}, \boldsymbol{Q}^{(d)}\right)}{\sum_{l \in \mathcal{L}} \mathrm{P}_{l}\left(\boldsymbol{P}^{(d)}, \boldsymbol{Q}^{(d)}, \boldsymbol{a}^{(d)}, \boldsymbol{b}^{(d)}\right)+\mathrm{P}_{C B S}\left(\boldsymbol{P}^{(d)}, \boldsymbol{Q}^{(d)}, \boldsymbol{a}^{(d)}, \boldsymbol{b}^{(d)}\right)}$ be the system EE at the $d$-th iteration of Algorithm 3. In fact, we can consider that $\left(\boldsymbol{P}^{(d)}, \boldsymbol{Q}^{(d)}, \boldsymbol{a}^{(d)}, \boldsymbol{b}^{(d)}\right)$ is the given block of resource allocation variables, $\eta^{(d)}$ is the system EE for such given resource allocation, and $\left(\boldsymbol{P}^{(d+1)}, \boldsymbol{Q}^{(d+1)}, \boldsymbol{a}^{(d+1)}, \boldsymbol{b}^{(d+1)}\right)$ is the updated block of resource allocation variables. We need to show that such updated block of resource allocation variables also improve system EE.

In the Step 3, Algorithm 3 updates power allocation variables while incorporating the assignment variables and system EE of the previous iteration in Algorithm 1. Because of the near-optimality of Algorithm 1, as demonstrated by Proposition 2, we obtain the following 
inequality.

$$
\begin{aligned}
& \sum_{j=1}^{K} \sum_{l=1}^{L} \sum_{n=1}^{N} a_{l, j}^{(d)} b_{n, l, j}^{(d)} \mathrm{T}_{j}^{(l, n)}\left(\boldsymbol{P}^{(d)}, \boldsymbol{Q}^{(d)}\right) \\
& \quad-\eta^{(d)}\left(\sum_{l \in \mathcal{L}} \mathrm{P}_{l}\left(\boldsymbol{P}^{(d)}, \boldsymbol{Q}^{(d)}, \boldsymbol{a}^{(d)}, \boldsymbol{b}^{(d)}\right)+\mathrm{P}_{C B S}\left(\boldsymbol{P}^{(d)}, \boldsymbol{Q}^{(d)}, \boldsymbol{a}^{(d)}, \boldsymbol{b}^{(d)}\right)\right) \\
& \leq \sum_{j=1}^{K} \sum_{l=1}^{L} \sum_{n=1}^{N} a_{l, j}^{(d)} b_{n, l, j}^{(d)} \mathrm{T}_{j}^{(l, n)}\left(\boldsymbol{P}^{(d+1)}, \boldsymbol{Q}^{(d+1)}\right) \\
& \quad-\eta^{(d)}\left(\sum_{l \in \mathcal{L}} \mathrm{P}_{l}\left(\boldsymbol{P}^{(d+1)}, \boldsymbol{Q}^{(d+1)}, \boldsymbol{a}^{(d)}, \boldsymbol{b}^{(d)}\right)+\mathrm{P}_{C B S}\left(\boldsymbol{P}^{(d+1)}, \boldsymbol{Q}^{(d+1)}, \boldsymbol{a}^{(d)}, \boldsymbol{b}^{(d)}\right)\right) .
\end{aligned}
$$

In the Step 4, Algorithm 3 updates the assignment variables while incorporating the updated power allocations and system EE of the previous iteration in Algorithm 2. As per Proposition 4, Algorithm 2 provides a near-optimal solution to P2.6. Consequently, we obtain the following inequality.

$$
\begin{aligned}
& \sum_{j=1}^{K} \sum_{l=1}^{L} \sum_{n=1}^{N} a_{l, j}^{(d)} b_{n, l, j}^{(d)} \mathrm{T}_{j}^{(l, n)}\left(\boldsymbol{P}^{(d+1)}, \boldsymbol{Q}^{(d+1)}\right) \\
& \quad-\eta^{(d)}\left(\sum_{l \in \mathcal{L}} \mathrm{P}_{l}\left(\boldsymbol{P}^{(d+1)}, \boldsymbol{Q}^{(d+1)}, \boldsymbol{a}^{(d)}, \boldsymbol{b}^{(d)}\right)+\mathrm{P}_{C B S}\left(\boldsymbol{P}^{(d+1)}, \boldsymbol{Q}^{(d+1)}, \boldsymbol{a}^{(d)}, \boldsymbol{b}^{(d)}\right)\right) \\
& \leq \sum_{j=1}^{K} \sum_{l=1}^{L} \sum_{n=1}^{N} a_{l, j}^{(d+1)} b_{n, l, j}^{(d+1)} \mathrm{T}_{j}^{(l, n)}\left(\boldsymbol{P}^{(d+1)}, \boldsymbol{Q}^{(d+1)}\right) \\
& \quad-\eta^{(d)}\left(\sum_{l \in \mathcal{L}} \mathrm{P}_{l}\left(\boldsymbol{P}^{(d+1)}, \boldsymbol{Q}^{(d+1)}, \boldsymbol{a}^{(d+1)}, \boldsymbol{b}^{(d+1)}\right)+\mathrm{P}_{C B S}\left(\boldsymbol{P}^{(d+1)}, \boldsymbol{Q}^{(d+1)}, \boldsymbol{a}^{(d+1)}, \boldsymbol{b}^{(d+1)}\right)\right) .
\end{aligned}
$$


Combining (A.8) and (A.9), we obtain

$$
\begin{aligned}
& \sum_{j=1}^{K} \sum_{l=1}^{L} \sum_{n=1}^{N} a_{l, j}^{(d+1)} b_{n, l, j}^{(d+1)} \mathrm{T}_{j}^{(l, n)}\left(\boldsymbol{P}^{(d+1)}, \boldsymbol{Q}^{(d+1)}\right) \\
& \quad-\eta^{(d)}\left(\sum_{l \in \mathcal{L}} \mathrm{P}_{l}\left(\boldsymbol{P}^{(d+1)}, \boldsymbol{Q}^{(d+1)}, \boldsymbol{a}^{(d+1)}, \boldsymbol{b}^{(d+1)}\right)+\mathrm{P}_{C B S}\left(\boldsymbol{P}^{(d+1)}, \boldsymbol{Q}^{(d+1)}, \boldsymbol{a}^{(d+1)}, \boldsymbol{b}^{(d+1)}\right)\right) \\
& \geq \sum_{j=1}^{K} \sum_{l=1}^{L} \sum_{n=1}^{N} a_{l, j}^{(d)} b_{n, l, j}^{(d)} \mathrm{T}_{j}^{(l, n)}\left(\boldsymbol{P}^{(d+1)}, \boldsymbol{Q}^{(d+1)}\right) \\
& \quad-\eta^{(d)}\left(\sum_{l \in \mathcal{L}} \mathrm{P}_{l}\left(\boldsymbol{P}^{(d+1)}, \boldsymbol{Q}^{(d+1)}, \boldsymbol{a}^{(d)}, \boldsymbol{b}^{(d)}\right)+\mathrm{P}_{C B S}\left(\boldsymbol{P}^{(d+1)}, \boldsymbol{Q}^{(d+1)}, \boldsymbol{a}^{(d)}, \boldsymbol{b}^{(d)}\right)\right)=0 .
\end{aligned}
$$

As per the Step 5 of Algorithm 3, we write

$$
\eta^{(d+1)}=\frac{\sum_{j=1}^{K} \sum_{l=1}^{L} \sum_{n=1}^{N} a_{l, j}^{(d+1)} b_{n, l, j}^{(d+1)} \mathrm{T}_{j}^{(l, n)}\left(\boldsymbol{P}^{(d+1)}, \boldsymbol{Q}^{(d+1)}\right)}{\sum_{l \in \mathcal{L}} \mathrm{P}_{l}\left(\boldsymbol{P}^{(d+1)}, \boldsymbol{Q}^{(d+1)}, \boldsymbol{a}^{(d+1)}, \boldsymbol{b}^{(d+1)}\right)+\mathrm{P}_{C B S}\left(\boldsymbol{P}^{(d+1)}, \boldsymbol{Q}^{(d+1)}, \boldsymbol{a}^{(d+1)}, \boldsymbol{b}^{(d+1)}\right)} .
$$

Combining (A.10) and (A.11), we obtain

$$
\begin{aligned}
& \left(\sum_{l \in \mathcal{L}} \mathcal{P}_{l}\left(\boldsymbol{P}^{(d+1)}, \boldsymbol{Q}^{(d+1)}, \boldsymbol{a}^{(d)}, \boldsymbol{b}^{(d)}\right)+\mathcal{P}_{C B S}\left(\boldsymbol{P}^{(d+1)}, \boldsymbol{Q}^{(d+1)}, \boldsymbol{a}^{(d)}, \boldsymbol{b}^{(d)}\right)\right)\left(\eta^{(d+1)}-\eta^{(d)}\right) \geq 0 \\
& \Longrightarrow \eta^{(d+1)} \geq \eta^{(d)} .
\end{aligned}
$$

Consequently, the updated block of resource allocation variables also improve system EE. In other words, Algorithm 3 obtains a sequence of EE where the system EE is monotonically increased at each iteration of Algorithm 3.

Since the system EE is upper bounded, the EE sequence obtained by Algorithm 3 must converge to a stable point. We need to justify that such a stable EE also satisfies the optimality condition, i.e. (10). For a proof of contradiction, we assume that the EE sequence obtained by Algorithm 3 converges to a point that does not satisfy (10) of our revised manuscript. In other words, $\lim _{d \rightarrow \infty} \eta^{(d)}=\eta^{(d+1)} \neq \eta$ where $\eta$ is the maximum achievable system EE. This leads to 
the following condition:

$$
\begin{aligned}
& \sum_{j=1}^{K} \sum_{l=1}^{L} \sum_{n=1}^{N} a_{l, j}^{(d+1)} b_{n, l, j}^{(d+1)} \mathrm{T}_{j}^{(l, n)}\left(\boldsymbol{P}^{(d+1)}, \boldsymbol{Q}^{(d+1)}\right) \\
& \quad-\eta^{(d)}\left(\sum_{l \in \mathcal{L}} \mathrm{P}_{l}\left(\boldsymbol{P}^{(d+1)}, \boldsymbol{Q}^{(d+1)}, \boldsymbol{a}^{(d+1)}, \boldsymbol{b}^{(d+1)}\right)+\mathrm{P}_{C B S}\left(\boldsymbol{P}^{(d+1)}, \boldsymbol{Q}^{(d+1)}, \boldsymbol{a}^{(d+1)}, \boldsymbol{b}^{(d+1)}\right)\right) \neq 0 \\
& \Longrightarrow \eta^{(d)} \neq \frac{\sum_{j=1}^{K} \sum_{l=1}^{L} \sum_{n=1}^{N} a_{l, j}^{(d+1)} b_{n, l, j}^{(d+1)} \mathrm{T}_{j}^{(l, n)}\left(\boldsymbol{P}^{(d+1)}, \boldsymbol{Q}^{(d+1)}\right)}{\sum_{l \in \mathcal{L}} \mathrm{P}_{l}\left(\boldsymbol{P}^{(d+1)}, \boldsymbol{Q}^{(d+1)}, \boldsymbol{a}^{(d+1)}, \boldsymbol{b}^{(d+1)}\right)+\mathrm{P}_{C B S}\left(\boldsymbol{P}^{(d+1)}, \boldsymbol{Q}^{(d+1)}, \boldsymbol{a}^{(d+1)}, \boldsymbol{b}^{(d+1)}\right)} .
\end{aligned}
$$

Using (A.11) and (A.13), we obtain $\eta^{(d)} \neq \eta^{(d+1)}$ which is contradictory to the assumption that the EE sequence obtained by Algorithm 3 converges. Therefore, the EE sequence obtained by Algorithm 3 must converge to a near-optimal system EE. This completes proof of the nearoptimality of Algorithm 3.

Near-optimality of Algorithm 4: As mentioned that the solutions to P2.2 and P2.3 converge when $\eta=0$. By plugging $\eta^{(d)}=0$ to (A.10), we obtain

$$
\sum_{j=1}^{K} \sum_{l=1}^{L} \sum_{n=1}^{N} a_{l, j}^{(d+1)} b_{n, l, j}^{(d+1)} \mathrm{T}_{j}^{(l, n)}\left(\boldsymbol{P}^{(d+1)}, \boldsymbol{Q}^{(d+1)}\right) \geq \sum_{j=1}^{K} \sum_{l=1}^{L} \sum_{n=1}^{N} a_{l, j}^{(d)} b_{n, l, j}^{(d)} \mathrm{T}_{j}^{(l, n)}\left(\boldsymbol{P}^{(d+1)}, \boldsymbol{Q}^{(d+1)}\right) .
$$

Eq. (A.14) is true for $\forall d$. Therefore, Algorithm 4 provides a sequence of non-decreasingly improved SE. Because of the total power constraints, the maximum achievable SE is upper bounded. Hence, Algorithm 4 must converge to a near-optimal SE. This completes proof of the near-optimality of Algorithm 4.

\section{APPENDIX G}

Since P3.1 provides a (local) optimal solution to P1, according to Lemma 1 , a solution to P3.1 is also weakly Pareto optimal to P0. We denote set of the weakly Pareto optimal solutions obtained from I-RSRM algorithm by $\mathcal{F}=\left\{f_{1}, f_{2}, \cdots f_{N_{F}}\right\}$. Notably, $f_{i}=\left\{f_{i}^{(1)}, f_{i}^{(2)}\right\}$ where $f_{i}^{(1)}$ and $f_{i}^{(2)}$ denote weakly Pareto optimal SE and EE obtained from the $i$-th solution of the I-RSRM algorithm, respectively, and $\mathcal{F} \subseteq \Psi$ where $\Psi$ is the optimal Paretofront of P0. We emphasize that no solution in the feasible space of P0 can dominate the following two EE-SE pairs, namely, $\left(\boldsymbol{S} \boldsymbol{E}\left(\boldsymbol{P}_{S E}, \boldsymbol{Q}_{S E}, \boldsymbol{a}_{S E}, \boldsymbol{b}_{S E}\right), \boldsymbol{E} \boldsymbol{E}\left(\boldsymbol{P}_{S E}, \boldsymbol{Q}_{S E}, \boldsymbol{a}_{S E}, \boldsymbol{b}_{S E}\right)\right)$ and $\left(\boldsymbol{S E}\left(\boldsymbol{P}_{E E}, \boldsymbol{Q}_{E E}, \boldsymbol{a}_{E E}, \boldsymbol{b}_{E E}\right), \boldsymbol{E} \boldsymbol{E}\left(\boldsymbol{P}_{E E}, \boldsymbol{Q}_{E E}, \boldsymbol{a}_{E E}, \boldsymbol{b}_{E E}\right)\right)$. Consequently, such two EE-SE pairs will be the two corner points of the optimal Pareto-front of P0. Meanwhile, in $\mathcal{F}, f_{1}$ and 
$f_{N_{F}}$ are obtained by setting $\epsilon=\boldsymbol{E} \boldsymbol{E}\left(\boldsymbol{P}_{S E}, \boldsymbol{Q}_{S E}, \boldsymbol{a}_{S E}, \boldsymbol{b}_{S E}\right)$ and $\epsilon=\boldsymbol{E} \boldsymbol{E}\left(\boldsymbol{P}_{E E}, \boldsymbol{Q}_{E E}, \boldsymbol{a}_{E E}, \boldsymbol{b}_{E E}\right)$, respectively. Thus, the corner points of $\mathcal{F}$ coincide with the corner points of the optimal Pareto-front. For the $i$-th non-corner point of $\mathcal{F}, \forall i, \boldsymbol{S E}\left(\boldsymbol{P}_{E E}, \boldsymbol{Q}_{E E}, \boldsymbol{a}_{E E}, \boldsymbol{b}_{E E}\right)<f_{i}^{(1)}<$ $\boldsymbol{S E}\left(\boldsymbol{P}_{S E}, \boldsymbol{Q}_{S E}, \boldsymbol{a}_{S E}, \boldsymbol{b}_{S E}\right)$ and $\boldsymbol{E} \boldsymbol{E}\left(\boldsymbol{P}_{S E}, \boldsymbol{Q}_{S E}, \boldsymbol{a}_{S E}, \boldsymbol{b}_{S E}\right)<f_{i}^{(2)}<\boldsymbol{E} \boldsymbol{E}\left(\boldsymbol{P}_{E E}, \boldsymbol{Q}_{E E}, \boldsymbol{a}_{E E}, \boldsymbol{b}_{E E}\right)$ are satisfied. Since each solution in $\mathcal{F}$ is non-dominated, $\forall i, f_{(i+1)}^{(1)}<f_{(i)}^{(1)}$ and $f_{(i)}^{(2)}<f_{(i+1)}^{(2)}$ are satisfied as well. Without loss of generality, let consider $\widehat{f}=\theta f_{i}+(1-\theta) f_{i+1}$ where $\theta \in(0,1)$. We can readily demonstrate that $f_{(i+1)}^{(1)}<\widehat{f}^{(1)}<f_{(i)}^{(1)}$ and $f_{(i)}^{(2)}<\widehat{f}^{(2)}<f_{(i+1)}^{(2)}$ are satisfied. Therefore, $\widehat{f}$ is non-dominated and it lies between the corner points of $\mathcal{F}$. Furthermore, EE and SE of $\widehat{f}$ can be achieved by using the resource allocations of $f_{i}$ and $f_{i+1}$ in a time-sharing manner. Hence, $\widehat{f}$ is also a weakly Pareto optimal solution to P0. Accordingly, by taking linear combination of the two consecutive points of $\mathcal{F}$, we obtain $\mathcal{F}^{(1)}$ as a new set of weakly Pareto optimal solutions to P0 where $\left|\mathcal{F}^{(1)}\right|-|\mathcal{F}|=N_{F}-1$. In this way, the set of achievable weakly Pareto optimal solutions can be evolved as $\mathcal{F} \rightarrow \mathcal{F}^{(1)} \rightarrow \mathcal{F}^{(2)} \rightarrow \cdots \rightarrow \mathcal{F}^{(\text {final })}$. In the aforementioned sequence, the number of weakly Pareto optimal solutions is increased at each evolution. As $\left|\mathcal{F}^{(\text {final })}\right|$ becomes sufficiently large, further evolution will not create distinct Pareto optimal EE and SE pairs. Essentially, the aforementioned sequence must converge to near-optimal Pareto-front, i.e., $\mathcal{F}^{(\text {final })} \approx \Psi$. In practice, such an $\mathcal{F}^{(\text {final })}$ set can be obtained by setting small value for the step size $\Delta$, i.e., $\Delta=\lim _{N_{F} \rightarrow \infty} \frac{\boldsymbol{E} \boldsymbol{E}\left(\boldsymbol{P}_{E E}, \boldsymbol{Q}_{E E}, \boldsymbol{a}_{E E}, \boldsymbol{b}_{E E}\right)-\boldsymbol{E} \boldsymbol{E}\left(\boldsymbol{P}_{S E}, \boldsymbol{Q}_{S E}, \boldsymbol{a}_{S E}, \boldsymbol{b}_{S E}\right)}{N_{F}}$ in the proposed I-RSRM algorithm. Evidently, I-RSRM can approach the near-optimal Pareto-front of P0. This completes the proof of Proposition 6.

\section{REFERENCES}

[1] Z. Zhao et al., "Federated-learning-enabled intelligent fog radio access networks: Fundamental theory, key techniques, and future trends," IEEE Wireless Commun., vol. 27, no. 2, pp. 22-28, Apr. 2020.

[2] M.-S. Al-Abiad et al. "Cross-layer cloud off-loading with quality of service guarantees in Fog-RANs," IEEE Trans. on Commun., vol. 67, no. 12, pp. 8435-8449, June 2019.

[3] M. Kaneko et al., "On the opportunities and challenges of NOMA-based fog radio access networks: An Overview," IEEE Access (accepted for publication).

[4] S. Cheng et al., "Vision, requirements, and technology trend of 6G: How to tackle the challenges of system coverage, capacity, user data-rate and movement speed," IEEE Wireless Commun., vol. 27, no. 2, pp. 218-228, Apr. 2020.

[5] D. Liu and C. Yang, "Energy efficiency of downlink networks with caching at base stations," IEEE J. Sel. Areas Commun., vol. 34, no. 4, pp. 907-922, Apr. 2016.

[6] Z. Zhao et al. "Cluster content caching: An energy-efficient approach to improve quality of service in cloud radio access networks," IEEE J. Sel. Areas Commun., vol. 34, no. 5, pp. 1207-1221, May 2016.

[7] M. Sheng et al., "Enhancement for content delivery with proximity communications in caching enabled wireless networks: Architecture and challenges," IEEE Commun. Mag., vol. 54, no. 8, pp. 70-76, Aug. 2016.

[8] L. Shi, L. Zhao, G. Zheng, Z. Han, and Y. Ye, "Incentive design for cache-enabled D2D underlaid cellular networks using Stackelberg game,” IEEE Trans. Veh. Technol., vol. 68, no. 1, pp. 765-779, Jan. 2019. 
[9] B. Chen, C. Yang, and A. F. Molisch, "Cache-enabled device-to-device communications: Offloading gain and energy cost," IEEE Trans. Wireless Commun., vol. 16, no. 7, pp. 4519-4536, Jul. 2017.

[10] Y. Shen et al., "Device-to-device-assisted communications in cellular networks: An energy efficient approach in downlink video sharing scenario," IEEE Trans. Wireless Commun., vol. 15, no. 2, pp. 1575-1587, Feb. 2016.

[11] Y. Xu, X. Li, and J. Zhang, "Device-to-device content delivery in cellular networks: Multicast or unicast," IEEE Trans. Veh. Technol., vol. 67, no. 5, pp. 4401-4414, May 2018.

[12] R. Amer, H. Elsawy, M. M. Butt, E. A. Jorswieck, M. Bennis, and N. Marchetti, "Optimizing joint probabilistic caching and communication for clustered D2D networks," [Online]. Available: https://arxiv.org/pdf/1810.05510.pdf.

[13] K. Kanevay, N. Aboutoraby, S. Sorour, and M. C. Reedy, "Cross-layer offloading in fog-RANs using device cooperation and network coding" IEEE Global Communication Workshops, Abu Dhabi, Dec. 2018, pp. 1-6.

[14] Y. Liu, Y. Wang, R. Sun, S. Meng, and R. Su, "Energy efficient downlink resource allocation for D2D-assisted cellular networks with mobile edge caching," IEEE Access, vol. 7, pp. 2053-2067, Jan. 2019.

[15] A. Douik, H. Dahrouj, O. Amin, B. AlOquibi, T. Y. A.-Naffouri, and M.-S. Alouini, "Mode selection and power allocation in multi-level cache-enabled networks," IEEE Communication Lett. (accepted for publication).

[16] H. Zhang, Y. Qiu, K. Long, G. K. Karagiannidis, X. Wang, and A. Nallanathan, "Resource allocation in NOMA-based fog radio access networks," IEEE Wireless Commun., vol. 25, no. 3, pp. 110-115, June 2018.

[17] I. Randrianantenaina, M. Kaneko, H. Dahrouj, H. ElSawy, and M.-S. Alouini, "Interference management in NOMA-based fog-radio access networks via Scheduling and power allocation," IEEE Trans. Commun. (accepted for publication).

[18] B. Liu et al., "Resource allocation for non-orthogonal multiple access-enabled fog radio access networks," IEEE Trans. Wireless Commun., vol. 19, no. 6, pp. 3867-3878, June 2020.

[19] X. Wen et al., "Interference pricing resource allocation and user-subchannel matching for NOMA hierarchy fog networks," IEEE J. Sel. Topics Sig. Proc., vol. 13, no. 3, pp. 467-479, June 2019.

[20] Z. Yan, M. Peng, and M. Daneshmand, "Cost-aware resource allocation for optimization of energy efficiency in fog radio access networks,” IEEE J. Sel. Areas Commun., vol. 36, no. 11, pp. 2581-2590, Oct. 2018.

[21] T. H. L. Dinh, M. Kaneko, E. H. Fukuday, and L. Boukhatem, "Energy efficient resource allocation optimization in fog radio access networks with outdated channel knowledge," IEEE Trans. Green Commun. Netw. (accepted for publication).

[22] Y. Mao, B. Clerckx, and V. Li, "Rate-splitting multiple access for downlink communication systems: Bridging, generalizing, and outperforming SDMA and NOMA," EURASIP J. Wireless Commun. and Network., no. 133, pp. 1-54, May 2018.

[23] B. Clerckx et al., "Rate-splitting unifying SDMA, OMA, NOMA, and multicasting in MISO broadcast channel: A simple two-user rate analysis," IEEE Wireless Commun. Lett., vol. 9, no. 3, pp. 349-353, Mar. 2020.

[24] H. Joudeh and B. Clerckx, "Robust transmission in downlink multiuser MISO systems: A rate-splitting approach," IEEE Trans. Signal Process., vol. 64, no. 23, pp. 6227-6242, Dec. 2016.

[25] D. Yu, J. Kim, and S.-H. Park, "An efficient rate-splitting multiple access scheme for the downlink of C-RAN systems," IEEE Commun. Lett., vol. 8, no. 6, pp. 1555-1558, Dec. 2019.

[26] Y. Mao, B. Clerckx, J. Zhang, V. O.K. Li, and M. Arafah, "Max-min fairness of $K$-user cooperative rate-splitting in MISO broadcast channel with user relaying," [Online]. Available: https://arxiv.org/pdf/1910.07843.pdf.

[27] W. Jaafar, S. Naser, S. Muhaidat, P.C. Sofotasios, H. Yanikomeroglu, "Multiple access in aerial networks: From orthogonal and non-orthogonal to rate-splitting,"[Online]. Available: https://arxiv.org/pdf/2005.13122.pdf.

[28] Y. Mao, B. Clerckx, and V. O. K. Li, "Rate-splitting for multi-antenna non-orthogonal unicast and multicast transmission: Spectral and energy efficiency analysis," IEEE Trans. Commun., vol. 67, no. 12, pp. 8754-8770, Dec. 2019.

[29] A. A. Ahmad, B. Matthiesen, A. Sezgin, and E. Jorswieck, "Energy efficiency in C-RAN using rate splitting and common message decoding," IEEE Int. Conf. Commun. Wrkshps., Dublin, June 2020, pp. 1-6.

[30] G. Zhou, Y. Mao, and B. Clerckx, "Rate-splitting multiple access for multi-antenna downlink communication systems: Spectral and energy efficiency tradeoff," [Online]. Available: https://arxiv.org/pdf/2001.03206.pdf. 
[31] Z. Yang et al., "Optimization of rate allocation and power control for rate splitting multiple access (RSMA)," [Online]. Available: https://arxiv.org/pdf/1903.08068.pdf.

[32] K.-M. Miettinen, Nonlinear Multi-objective Optimization, Kluwer Academic Publishers, Boston, 1999.

[33] Z.-Q. Luo, and S. Zhang, "Dynamic spectrum management: Complexity and duality,” IEEE J. Sel. Areas Commun., vol. 2, no. 1, pp. 57-73, Feb. 2008.

[34] A. Mesodiakaki et al., "Energy and spectrum efficient user association in millimeter wave backhaul small cell networks," IEEE Trans. Veh. Technol., vol. 66, no. 2 pp. 1810-1821, Feb. 2017.

[35] C. Ng AND D. S. Hirshchberg, "Three-dimensional stable matching problems," SIAM J. Disc. Math., vol. 4, no. 2, pp. 245-252, May 1991.

[36] 3GPP, TR 36.819 (V9.0.0), “Further advancement for E-UTRA physical layer aspects (Release 9)," Mar. 2010.

[37] F. Fang, J. Cheng, and Z. Ding, "Joint energy efficient subchannel and power optimization for a downlink NOMA heterogeneous network," IEEE Trans. Veh. Technol., vol. 68, no. 2, pp. 1351-1364, Feb. 2019.

[38] M. Z. Hassan et al., "Device-clustering and rate-splitting enabled device-to-device cooperation framework in fog radio access network," [Online]. Available: https://arxiv.org/pdf/2007.03827.pdf.

[39] A. Douik, H. Dahrouj, O. Amin, B. AlOquibi, T. Y. A.-Naffouri, and M.-S. Alouini, "Mode selection and power allocation in multi-level cache-enabled networks," IEEE Communication Lett. (accepted for publication).

[40] Z. Han et al., Game Theory in Wireless and Communication Networks: Theory, Models and Applications, Cambridge, UK: Cambridge University Press, 2011.

[41] J. Yuan, Z. Li, W. Yu, and B. Li, "A cross-layer optimization framework for multicast in multi-hop wireless networks," Proc. IEEE 1st Int. Conf. Wireless Internet, Budapest, pp. 47-54, 2005. 